\title{
Kondo lattice model: Unitary transformations, spin dynamics, strongly correlated charged modes, and vacuum instability.
}

\author{
J.M. Prats and F. López-Aguilar \\ Departament de Física, Grup d'Electromagnetisme, Universitat Autònoma de Barcelona, \\ Bellaterra, E-08193 Barcelona, Spain
}

\begin{abstract}
Using unitary transformations, we express the Kondo lattice Hamiltonian in terms of fermionic operators that annihilate the ground state of the interacting system and that represent the best possible approximations to the actual charged excitations. In this way, we obtain an effective Hamiltonian which, for small couplings, consists in a kinetic term for conduction electrons and holes, an RKKY-like term, and a renormalized Kondo interaction. The physical picture of the system implied by this formalism is that of a vacuum state consisting in a background of RKKY-induced spin correlations, where two kinds of elementary modes can be excited: Soft neutral modes associated with deformations of the spin liquid, which lead to very large low-temperature values of the heat capacity and magnetic susceptibility, and charged modes corresponding to the excitation of electrons and holes in the system. Using the translational and spin rotational symmetries, we construct a simple ansatz to determine the charged excitations neglecting the effects of the spin correlations. Apart from the 'normal', uncorrelated states, we find strongly correlated charged modes involving soft electrons (or holes) and spin fluctuations, which strongly renormalize the lowenergy charged spectrum, and whose energy becomes negative beyond a critical coupling, signaling a vacuum instability and a transition to a new phase.
\end{abstract}

PACS: 71.27.+a, 75.30.Mb, 75.20.Hr. 


\section{INTRODUCTION}

Despite many years of intense experimental and theoretical studies of heavy-fermion (HF) systems, 1 their physical picture still remains controversial. It is generally believed that both HF metals and the so-called mixed-valence compounds are well-represented by the Anderson lattice model (ALM) and that their phenomenologies strongly depend on the location of the $\mathrm{f}$ band with respect to the Fermi level. Ce-based HF compounds are usually regarded as an extreme case in which the deep $f$ level and high on-site Coulomb repulsion strongly inhibit f-charge fluctuations. This has led many authors to regard the Kondo lattice model (KLM) as a canonical model for these compounds since it has been proved to be the limit of the ALM for vanishing f-charge fluctuations, 5 and it exclusively involves the degrees of freedom that are expected to be relevant to the low-energy phenomenology of these materials.

Among the most important features characterizing this phenomenology are the very large values of the low-temperature magnetic susceptibility and linear coefficient of the specific heat, the appearance, in some cases, of antiferromagnetic order with strongly quenched local moments, and the transition to a superconducting phase in systems like $\mathrm{CeCu}_{2} \mathrm{Si}_{2}$.

HF metals are often regarded as 'dense Kondo systems' and its properties qualitatively discussed extending to the lattice the results obtained for the single 6 目 and two-impurity 8 cases. In the first of these cases (Kondo problem), a singlet state is formed at zero temperature between the spins of the impurity and of the conduction electrons. 9 Since the low-energy singlet-triplet excitation associated with this (Abrikosov-Shul) resonance gives a contribution to the specific heat, 10 the formation of one of such resonances at each site of a Kondo lattice would account for the very high heat capacities measured in these systems. In the two-impurity case, however, we have an additional ingredient, which is the appearance of an induced, inter-site (RKKY) interaction. Thus, magnetic order in the Kondo lattice is often interpreted as due to a competition between the RKKY interaction (which tends to produce magnetic order) and the Kondo effect (which tends to quench the local moments) 11 
These interpretations, however, have been criticized by Nozières, 12 who pointed out that only a very narrow layer of conduction electrons around the Fermi level are energetically capable of forming Kondo singlets, which implies that we can have only one 'efficient' electron per many local spins. Moreover, Nozières argument implies that, if strongly correlated (SC) states between conduction electrons and local moments are formed in the system, they should not correspond to local Kondo singlets but to extended, collective states in which one electron couples to many local moments, namely, to a spin wave. The calculation of such SC states or 'magnetic polarons' is, in fact, one of the main objectives of this article.

Another consequence of the Nozières argument is that only the very-low-energy part of the electronic dispersion relation can be strongly renormalized and, obviously, this mass renormalization cannot account for a measured entropy at the characteristic Kondo lattice temperature $\left(T_{K L}\right)$ of the order of $N \ln L$, where $L$ and $N$ are the f-level degeneracy and number of lattice sites respectively. Since conduction electrons are the only source of electric charge in the KLM, charged fermions cannot be responsible for the huge masses implied by the low-temperature specific heat and magnetic susceptibility.

In contrast to the slave-boson 13,44 and variational 15 approximations, which defend the existence of charged heavy particles, the above arguments have led Kagan, Kikoin, and Prokof'ev 16 to suggest that the main contribution to the low-temperature thermodynamics of these materials comes from neutral Fermi excitations of spin origin. They show 6 that this picture is in good agreement with the available experimental data while a charged-fermionbased approach seems to be in conflict with De Haas-Van Alphen experiments.17

In this paper, we introduce a mathematical formalism to study the KLM that leads to a physical picture of HF systems (summarized in the conlusions) which does not enter in conflict with the Nozières exhaustion problem and which, among other things, supports the views presented in Ref. 16.

The basic idea of this formalism is presented in Section II, and consists in carrying out a unitary transformation to determine a new set of canonical fermionic operators that annihilate the ground state of the interacting system. We find that, when the Kondo lattice 
Hamiltonian (KLH) is expressed in terms of these operators, it essentially consists, for small couplings, in a kinetic term for electrons and holes containing a gap in its dispersion relation, an RKKY term, and a Kondo interaction in which the terms involving an electron and a hole operator have been cancelled.

Using this expression, we discuss magnetic order in Section III. We argue that, since all the terms of $H$ that contain fermions annihilate the vacuum state determined in Section II, the RKKY interaction is the only one responsible for the dynamics of the spin lattice unless some electronic instability (which we indeed find for stronger couplings) takes place. Thus, we believe that the reasons for an eventual formation of a spin liquid should be seeked in the enhancement of spin fluctuations due to the strong frustration produced by the long-range RKKY interaction. We also argue, in agreement with Ref. 16, that the very large specific heat measured in these systems should be attributed to the enormous entropy increase associated to the thermal breakdown of soft spin correlations.

We mentioned that the dispersion relation corresponding to the kinetic term for electrons and holes of the transformed Hamiltonian contains a gap. However, if no symmetry is broken and no bound states are formed, there is no physical reason for the appearance of a gap in the middle of the conduction band. In Section IV, we calculate the actual (uncorrelated) charged modes neglecting the effect of the spin correlations and show, to leading order, that no gap is actually opened at the Fermi level.

As we mentioned when we discussed the Nozières exhaustion problem, if SC states are formed in the system, they should be extended, collective states in which a conduction electron or hole couples to a spin wave. In Section V, we look for such states. We argue that, to include the effect of the high-energy tail of the Kondo interaction, these modes should be constructed from 'optimal' fermionic operators that represent the best possible approximations to the actual (uncorrelated) charged modes. These new operators are determined with the aid of a second unitary transformation that leaves the ground state invariant and which is determined variationally. We find that the expression of $H$ in terms of this new set of canonical operators essentially consists in a kinetic term containing a much smaller gap, 
an RKKY term without any modification, and a residual Kondo interaction which is only effective at low energies. With these optimal operators and neglecting the spin correlations, we construct SC charged modes using an ansatz that exploits all the symmetries of the Hamiltonian. We find that SC modes of spin $3 / 2$ and $1 / 2$ can form, respectively, in ferro and antiferromagnetic Kondo lattices and that its formation is much more favored in the last case. We also find that for suffiently strong couplings, these modes must strongly renormalize the low-energy charged spectrum and that, beyond a critical coupling, they would condensate in the ground state producing an instability and, consequently, a transition to a new phase. Since the coupling of spin waves to conduction electrons to form SC modes influences the spin correlations (which are the main source of low-energy entropy), this phase transition should be accompanied by important changes in the specific heat.

\section{FIRST TRANSFORMATION: INTERACTING VACUUM STATE}

We shall study a KLM consisting in a conduction band coupled to a lattice of $s=1 / 2$ local moments by an exchange interaction. The Hamiltonian is given by

$$
H=\sum_{\mathbf{k}, \alpha} \varepsilon_{\mathbf{k}} c_{\mathbf{k} \alpha}^{\dagger} c_{\mathbf{k} \alpha}+J \sum_{i} \mathbf{S}_{e i} \mathbf{S}_{f i}
$$

where $\mathbf{S}_{e i}$ and $\mathbf{S}_{f i}$ are the spin of the conduction electrons and the local spin at site $i$ respectively. $\mathbf{S}_{e i}=1 / 2 \sum_{\alpha, \beta} c_{i \alpha}^{\dagger} \sigma_{\alpha \beta} c_{i \beta}$, where $c_{i \alpha}^{\dagger}$ are electronic operators in the site (Wannier) representation. If $\mathbf{R}_{i}$ is the position of the $i$ site, these operators are given, for a lattice with $N$ sites, by $c_{i \alpha}^{\dagger}=N^{-1 / 2} \sum_{\mathbf{k}} \mathrm{e}^{-i \mathbf{R}_{i} \mathbf{k}} c_{\mathbf{k} \alpha}^{\dagger}$.

We will consider the case of a half-filled conduction band with a symmetric density of states. Although the symmetry between electrons and holes will be very convenient to simplify the formalism, the scenario presented in this paper is expected to hold in all situations around half filling. To simplify the notation, we shall take energy units in which the band width is normalized ( $W=1)$. The final formulas that we will obtain can, of course, be written in arbitrary units by suitably reintroducing $W$ using dimensional analysis. In (I), 
we have already subtracted the chemical potential in $\varepsilon_{\mathbf{k}}$ and, thus, $W=1$ and the electronhole symmetry, imply that $-1 / 2 \leq \varepsilon_{\mathbf{k}} \leq 1 / 2$. Finally, to enable an analytic calculation of many quantities throughout this paper, we will assume a constant density of states $D$. $D$ normalized and constant, and $W=1$ imply $D=1$.

The fundamental operators $c_{\mathbf{k} \alpha}, \mathbf{S}_{f i}$ are characterized by the following algebraic properties:

$$
\begin{aligned}
\left\{c_{\mathbf{k} \alpha}, c_{\mathbf{k}^{\prime} \beta}^{\dagger}\right\} & =\delta_{\mathbf{k k}^{\prime}} \delta_{\alpha \beta},\left\{c_{\mathbf{k} \alpha}, c_{\mathbf{k}^{\prime} \beta}\right\}=0, \\
{\left[S_{f i}^{\alpha}, S_{f j}^{\beta}\right] } & =i \delta_{i j} \sum_{\gamma} \epsilon_{\alpha \beta \gamma} S_{f i}^{\gamma}, \\
\mathbf{S}_{f i}^{\dagger} & =\mathbf{S}_{f i}, \\
\mathbf{S}_{f i}^{2} & =3 / 4, \\
{\left[c_{\mathbf{k} \alpha}, \mathbf{S}_{f i}\right] } & =0 .
\end{aligned}
$$

As it is well-known, Eqs. (3) and (4) can be satisfied by writing $\mathbf{S}_{f i}=1 / 2 \sum_{\alpha, \beta} f_{i \alpha}^{\dagger} \sigma_{\alpha \beta} f_{i \beta}$, where $f_{i \alpha}^{\dagger}$ and $f_{i \alpha}$ are creation and annihilation operators of $f$ electrons satisfying canonical anticommutation relations. It can be proved (and is physically obvious) that the condition (5) is equivalent to demanding that each site be occupied by one and only one $f$ electron. As it will soon become clear, instead of using the $\mathbf{S}_{f i}$ operators, it is much more convenient to work with the following equivalent set of operators:

$$
\begin{aligned}
s_{1, i} & \equiv \sqrt{2}\left(S_{f i}^{x}+i S_{f i}^{y}\right)=\sqrt{2} f_{i \uparrow}^{\dagger} f_{i \downarrow}, \\
s_{0, i} & \equiv 2 S_{f i}^{z}=f_{i \uparrow}^{\dagger} f_{i \uparrow}-f_{i \downarrow}^{\dagger} f_{i \downarrow}, \\
s_{-1, i} & \equiv \sqrt{2}\left(S_{f i}^{x}-i S_{f i}^{y}\right)=\sqrt{2} f_{i \downarrow}^{\dagger} f_{i \uparrow} .
\end{aligned}
$$

It is straightforward to see that these operators, acting on states containing a single $f$ electron per lattice site, satisfy the following multiplication table:

$$
\begin{aligned}
s_{0, i} s_{1, i} & =s_{1, i} & s_{0, i} s_{-1, i} & =-s_{-1, i} \\
s_{1, i} s_{0, i} & =-s_{1, i} & s_{-1, i} s_{0, i} & =s_{-1, i} \\
s_{1, i} s_{-1, i} & =1+s_{0, i} & s_{1, i}^{2} & =s_{-1, i}^{2}=0 \\
s_{-1, i} s_{1, i} & =1-s_{0, i} & s_{0, i}^{2} & =1
\end{aligned}
$$


as well as the properties

$$
\begin{gathered}
s_{1, i}^{\dagger}=s_{-1, i}, s_{0, i}^{\dagger}=s_{0, i}, \\
{\left[s_{l, i}, s_{l^{\prime}, j}\right]=0 \quad \forall i \neq j .}
\end{gathered}
$$

Eqs. (8)-(10) can also be regarded as the defining conditions of the algebra of local moments since, as it can be readily checked, they imply Eqs. (3)-(5).

The multiplication table (8) will be extremely useful since it will enable us to reduce products of $s_{l}$ operators corresponding to the same lattice site. For example,

$$
s_{0,5} s_{-1,7} s_{1,5} s_{1,7}=s_{1,5}-s_{1,5} s_{0,7}
$$

In order to exploit the symmetry of the Hamiltonian (1) under lattice translations, it is convenient to work with all operators in the momentum representation:

$$
c_{\mathbf{k} \alpha}^{\dagger}=N^{-1 / 2} \sum_{i} \mathrm{e}^{i \mathbf{R}_{i} \mathbf{k}} c_{i \alpha}^{\dagger}, \quad s_{l, \mathbf{k}}=N^{-1 / 2} \sum_{i} \mathrm{e}^{i \mathbf{R}_{i} \mathbf{k}} s_{l, i}
$$

where $\mathbf{k}$ is always in the first Brillouin zone. The $s_{l, \mathbf{k}}$ operators satisfy the following equations, which will later be of much use:

$$
\begin{aligned}
{\left[s_{0, \mathbf{k}}, s_{ \pm 1, \mathbf{k}^{\prime}}\right] } & = \pm 2 N^{-1 / 2} s_{ \pm 1, \mathbf{k}+\mathbf{k}^{\prime}} \\
{\left[s_{1, \mathbf{k}}, s_{-1, \mathbf{k}^{\prime}}\right] } & =2 N^{-1 / 2} s_{0, \mathbf{k}+\mathbf{k}^{\prime}} \\
{\left[s_{l, \mathbf{k}}, s_{l, \mathbf{k}^{\prime}}\right] } & =0 \\
s_{l, \mathbf{k}}^{\dagger} & =s_{-l,-\mathbf{k}} .
\end{aligned}
$$

Another tool which we are going to use constantly is the development of a product of two $s_{l}$ operators in the momentum representation; here is an example:

$$
s_{-1, \mathbf{k}} s_{1, \mathbf{k}^{\prime}}=\delta\left(\mathbf{k}+\mathbf{k}^{\prime}\right)-N^{-1 / 2} s_{0, \mathbf{k}+\mathbf{k}^{\prime}}+N^{-1} \sum_{i \neq j} \mathrm{e}^{i\left(\mathbf{R}_{i} \mathbf{k}+\mathbf{R}_{j} \mathbf{k}^{\prime}\right)} s_{-1, i} s_{1, j}
$$

Finally, in the momentum representation, the KLH (1) is given by 


$$
H=\varepsilon_{\mathbf{k}} c_{\mathbf{k} \alpha}^{\dagger} c_{\mathbf{k} \alpha}+\frac{J}{4 N^{1 / 2}}\left[\left(c_{\mathbf{k} \uparrow}^{\dagger} c_{\mathbf{k}^{\prime} \uparrow}-c_{\mathbf{k} \downarrow}^{\dagger} c_{\mathbf{k}^{\prime} \downarrow}\right) s_{0, \mathbf{k}^{\prime}-\mathbf{k}}+\sqrt{2} c_{\mathbf{k} \uparrow}^{\dagger} c_{\mathbf{k}^{\prime} \downarrow} s_{-1, \mathbf{k}^{\prime}-\mathbf{k}}+\sqrt{2} c_{\mathbf{k} \downarrow}^{\dagger} c_{\mathbf{k}^{\prime} \uparrow} s_{1, \mathbf{k}^{\prime}-\mathbf{k}}\right],
$$

where, as in many equations throughout this paper, sum over repeated indexes in each term of the equation is implicitly understood.

We want to study this Hamiltonian for small values of $J / W$, which is the region characteristic of HF systems. In the absence of interaction, all the spin configurations will be degenerate, and the ground (or vacuum) state of the system $\left|\Phi_{0}\right\rangle$ will consist in a completely disordered spin lattice plus a Fermi sea containing all electrons with negative energy. The charged excitations of this system are electrons and holes consisting, respectively, in adding or removing an electron from this Fermi sea. To simplify the notation, $\mathbf{q}(\mathbf{p})$ will always denote a wave vector located below (above) the Fermi surface $\left(\varepsilon_{\mathbf{q}}<0, \varepsilon_{\mathbf{p}}>0\right)$, while $\mathbf{k}$ will be a wave vector without such restrictions. As it is obvious and well-known, the creation and annihilation operators corresponding to electrons and holes are given by

$$
e_{\mathbf{p} \alpha}^{\dagger}=c_{\mathbf{p} \alpha}^{\dagger}, e_{\mathbf{p} \alpha}=c_{\mathbf{p} \alpha}, h_{\mathbf{q} \alpha}^{\dagger}=c_{-\mathbf{q},-\alpha}, h_{\mathbf{q} \alpha}=c_{-\mathbf{q},-\alpha}^{\dagger}
$$

and the non-interacting vacuum state can be written as

$$
\left|\Phi_{0}\right\rangle=\sum_{\alpha_{i}=\uparrow, \downarrow} C_{\alpha_{1}, \ldots, \alpha_{N}}^{(0)} f_{1 \alpha_{1}}^{\dagger} \ldots f_{N \alpha_{N}}^{\dagger}\left|\Psi_{0}\right\rangle,
$$

where $\left|\Psi_{0}\right\rangle$ is a state annihilated by $e_{\mathbf{p} \alpha}$ and $h_{\mathbf{q} \alpha}$, and the coefficients $C_{\alpha_{1}, \ldots, \alpha_{N}}^{(0)}$ are given by $C_{\alpha_{1}, \ldots, \alpha_{N}}^{(0)}=2^{-N / 2} \mathrm{e}^{i R_{\alpha_{1} \ldots \alpha_{N}}}$, where $R_{\alpha_{1} \ldots \alpha_{N}}$ are random phases, as it corresponds to a completely disordered spin lattice.

The KLH (16) is given, in terms of the operators (17), by

$$
\begin{aligned}
& H=\left(\sum_{q} 2 \varepsilon_{\mathbf{q}}\right)+\varepsilon_{\mathbf{p}} e_{\mathbf{p} \alpha}^{\dagger} e_{\mathbf{p} \alpha}-\varepsilon_{\mathbf{q}} h_{\mathbf{q} \alpha}^{\dagger} h_{\mathbf{q} \alpha} \\
& +\frac{J}{4 N^{1 / 2}}\left[\left(e_{\mathbf{p} \uparrow}^{\dagger} e_{\mathbf{p}^{\prime} \uparrow}-e_{\mathbf{p} \downarrow}^{\dagger} e_{\mathbf{p}^{\prime} \downarrow}\right) s_{0, \mathbf{p}^{\prime}-\mathbf{p}}+\sqrt{2} e_{\mathbf{p} \uparrow}^{\dagger} e_{\mathbf{p}^{\prime} \downarrow} s_{-1, \mathbf{p}^{\prime}-\mathbf{p}}+\sqrt{2} e_{\mathbf{p} \downarrow}^{\dagger} e_{\mathbf{p}^{\prime} \uparrow} s_{1, \mathbf{p}^{\prime}-\mathbf{p}}\right] \\
& +\frac{J}{4 N^{1 / 2}}\left[\left(h_{\mathbf{q} \uparrow}^{\dagger} h_{\mathbf{q}^{\prime} \uparrow}-h_{\mathbf{q}_{\downarrow}}^{\dagger} h_{\mathbf{q}^{\prime} \downarrow}\right) s_{0, \mathbf{q}^{\prime}-\mathbf{q}}-\sqrt{2} h_{\mathbf{q} \uparrow}^{\dagger} h_{\mathbf{q}^{\prime} \downarrow} s_{-1, \mathbf{q}^{\prime}-\mathbf{q}}-\sqrt{2} h_{\mathbf{q}_{\downarrow}}^{\dagger} h_{\mathbf{q}^{\prime} \uparrow} s_{1, \mathbf{q}^{\prime}-\mathbf{q}}\right] \\
& +\frac{J}{4 N^{1 / 2}}\left[\left(e_{\mathbf{p} \uparrow}^{\dagger} h_{\mathbf{q} \downarrow}^{\dagger}-e_{\mathbf{p} \downarrow}^{\dagger} h_{\mathbf{q} \uparrow}^{\dagger}\right) s_{0,-\mathbf{q}-\mathbf{p}}+\sqrt{2} e_{\mathbf{p} \uparrow}^{\dagger} h_{\mathbf{q} \uparrow}^{\dagger} s_{-1,-\mathbf{q}-\mathbf{p}}+\sqrt{2} e_{\mathbf{p} \downarrow}^{\dagger} h_{\mathbf{q} \downarrow}^{\dagger} s_{1,-\mathbf{q}-\mathbf{p}}\right] \\
& +\frac{J}{4 N^{1 / 2}}\left[\left(h_{\mathbf{q} \downarrow} e_{\mathbf{p} \uparrow}-h_{\mathbf{q} \uparrow} e_{\mathbf{p} \downarrow}\right) s_{0, \mathbf{q}+\mathbf{p}}+\sqrt{2} h_{\mathbf{q} \uparrow} e_{\mathbf{p} \uparrow} s_{1, \mathbf{q}+\mathbf{p}}+\sqrt{2} h_{\mathbf{q} \downarrow} e_{\mathbf{p} \downarrow} s_{-1, \mathbf{q}+\mathbf{p}}\right] .
\end{aligned}
$$


This expression makes clear that $\left|\Phi_{0}\right\rangle$ is not an eigenstate (and, hence, not the ground state) of $H$ when $J \neq 0$, because the terms (22) do not annihilate $\left|\Phi_{0}\right\rangle$ but create additional electron-hole excitations accompanied by a spin fluctuation.

To determine the elementary excitations of the interacting system, we must first find the interacting vacuum state $|\Phi\rangle$. Our approach will consist in determining a unitary transformation that maps the basic operators $c_{\mathbf{k} \alpha}, s_{l, \mathbf{k}}$ into a new set $\tilde{c}_{\mathbf{k} \alpha}, \tilde{s}_{l, \mathbf{k}}$ such that $|\Phi\rangle$ can be written as

$$
|\Phi\rangle=\sum_{\alpha_{i}=\uparrow, \downarrow} C_{\alpha_{1}, \ldots, \alpha_{N}} \tilde{f}_{1 \alpha_{1}}^{\dagger} \ldots \tilde{f}_{N \alpha_{N}}^{\dagger}|\Psi\rangle
$$

where $\tilde{e}_{\mathbf{p} \alpha}|\Psi\rangle=\tilde{h}_{\mathbf{q} \alpha}|\Psi\rangle=0$, and the new local Fermi operators $\tilde{f}_{i, \alpha}$ are related to $\tilde{s}_{l, i}$ by Eq.

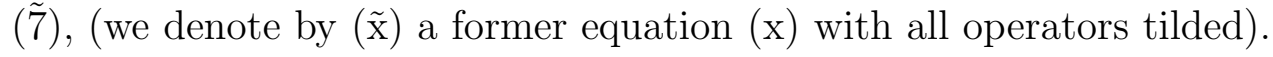

The transformation is chosen to be unitary to ensure that the new operators continue satisfying the basic, defining algebra (2/i) -(扄). The relationship between the initial and transformed operators is, thus,

$$
c_{\mathbf{k} \alpha}^{\dagger}=\mathrm{e}^{\tilde{T}} \tilde{c}_{\mathbf{k} \alpha}^{\dagger} \mathrm{e}^{-\tilde{T}}, s_{l, \mathbf{k}}=\mathrm{e}^{\tilde{T}} \tilde{s}_{l, \mathbf{k}} \mathrm{e}^{-\tilde{T}}, \quad \tilde{T}^{\dagger}=-\tilde{T} .
$$

Since we are interested, at this point, in determining a ground state that does not break any symmetry, we will demand that $\tilde{T}$ preserve the charge, lattice translational, and spin rotational symmetries. The simplest operator involving fermions that satisfies this condition is

$$
\tilde{T}=\frac{J}{4 N^{1 / 2}} \tilde{T}\left(\mathbf{k}, \mathbf{k}^{\prime}\right)\left[\left(\tilde{c}_{\mathbf{k} \uparrow}^{\dagger} \tilde{c}_{\mathbf{k}^{\prime} \uparrow}-\tilde{c}_{\mathbf{k} \downarrow}^{\dagger} \tilde{c}_{\mathbf{k}^{\prime} \downarrow}\right) \tilde{s}_{0, \mathbf{k}^{\prime}-\mathbf{k}}+\sqrt{2} \tilde{c}_{\mathbf{k} \uparrow}^{\dagger} \tilde{c}_{\mathbf{k}^{\prime} \downarrow} \tilde{s}_{-1, \mathbf{k}^{\prime}-\mathbf{k}}+\sqrt{2} \tilde{c}_{\mathbf{k} \downarrow}^{\dagger} \tilde{c}_{\mathbf{k}^{\prime} \uparrow} \tilde{s}_{1, \mathbf{k}^{\prime}-\mathbf{k}}\right]
$$

where

$$
\tilde{T}^{\star}\left(\mathbf{k}^{\prime}, \mathbf{k}\right)=-\tilde{T}\left(\mathbf{k}, \mathbf{k}^{\prime}\right)
$$

in order to satisfy $\tilde{T}^{\dagger}=-\tilde{T}$. Apart from this condition, the function $\tilde{T}\left(\mathbf{k}, \mathbf{k}^{\prime}\right)$ is arbitrary, and it will be determined by demanding that the state (24) be the actual interacting vacuum state. 
For small values of $J$, the new operators are expected to be slight deformations of the initial ones. Thus, we will expand Eq. 25) in powers of $\tilde{T}$, retaining the first order terms only:

$$
c_{\mathbf{k} \alpha}^{\dagger}=\tilde{c}_{\mathbf{k} \alpha}^{\dagger}+\left[\tilde{T}, \tilde{c}_{\mathbf{k} \alpha}^{\dagger}\right], s_{l, \mathbf{k}}=\tilde{s}_{l, \mathbf{k}}+\left[\tilde{T}, \tilde{s}_{l, \mathbf{k}}\right]
$$

These equations are the basis of the forecoming calculations. Using Eqs. (2) and (13), we can write them in a more explicit form:

$$
\begin{array}{r}
c_{\mathbf{k} \uparrow(\downarrow)}^{\dagger}=\tilde{\alpha}(\mathbf{k})\left\{\tilde{c}_{\mathbf{k} \uparrow(\downarrow)}^{\dagger}+\frac{J}{4 N^{1 / 2}} \tilde{T}\left(\mathbf{k}^{\prime}, \mathbf{k}\right)\left[+(-) \tilde{c}_{\mathbf{k}^{\prime} \uparrow(\downarrow)}^{\dagger} \tilde{s}_{0, \mathbf{k}-\mathbf{k}^{\prime}}+\sqrt{2} \tilde{c}_{\mathbf{k}^{\prime} \downarrow(\uparrow)}^{\dagger} \tilde{s}_{1(-1), \mathbf{k}-\mathbf{k}^{\prime}}\right]\right\}, \\
c_{\mathbf{k} \uparrow(\downarrow)}=\tilde{\alpha}(\mathbf{k})\left\{\tilde{c}_{\mathbf{k} \uparrow(\downarrow)}+\frac{J}{4 N^{1 / 2}} \tilde{T}^{\star}\left(\mathbf{k}^{\prime}, \mathbf{k}\right)\left[+(-) \tilde{c}_{\mathbf{k}^{\prime} \uparrow(\downarrow)} \tilde{s}_{0, \mathbf{k}^{\prime}-\mathbf{k}}+\sqrt{2} \tilde{c}_{\mathbf{k}^{\prime} \downarrow(\uparrow)} \tilde{s}_{-1(1), \mathbf{k}^{\prime}-\mathbf{k}}\right]\right\}, \\
s_{0, \mathbf{k}}=\tilde{s}_{0, \mathbf{k}}+\frac{J}{2 N} \tilde{T}\left(\mathbf{k}_{\mathbf{1}}, \mathbf{k}_{\mathbf{2}}\right)\left[\sqrt{2} \tilde{c}_{\mathbf{k}_{\mathbf{1}} \uparrow}^{\dagger} \tilde{c}_{\mathbf{k}_{\mathbf{2}} \downarrow} \tilde{s}_{-1, \mathbf{k}_{\mathbf{2}}-\mathbf{k}_{\mathbf{1}}+\mathbf{k}}-\sqrt{2} \tilde{c}_{\mathbf{k}_{\mathbf{1}} \downarrow}^{\dagger} \tilde{c}_{\mathbf{k}_{\mathbf{2}} \uparrow} \tilde{s}_{\left.1, \mathbf{k}_{\mathbf{2}}-\mathbf{k}_{\mathbf{1}}+\mathbf{k}\right]}\right], \\
s_{1(-1), \mathbf{k}}=\tilde{s}_{1(-1), \mathbf{k}}+(-) \frac{J}{2 N} \tilde{T}\left(\mathbf{k}_{\mathbf{1}}, \mathbf{k}_{\mathbf{2}}\right)\left[\left(\tilde{c}_{\mathbf{k}_{\mathbf{1}} \uparrow}^{\dagger} \tilde{c}_{\mathbf{k}_{\mathbf{2}} \uparrow}-\tilde{c}_{\mathbf{k}_{\mathbf{1}} \downarrow}^{\dagger} \tilde{c}_{\mathbf{k}_{\mathbf{2}} \downarrow}\right) \tilde{s}_{1(-1), \mathbf{k}_{\mathbf{2}}-\mathbf{k}_{\mathbf{1}}+\mathbf{k}}\right. \\
\left.-\sqrt{2} \tilde{c}_{\mathbf{k}_{1} \uparrow(\downarrow)}^{\dagger} \tilde{c}_{\mathbf{k}_{\mathbf{2}} \downarrow(\uparrow)} \tilde{s}_{\left.0, \mathbf{k}_{\mathbf{2}}-\mathbf{k}_{\mathbf{1}}+\mathbf{k}\right]}\right],
\end{array}
$$

where the coefficient $\tilde{\alpha}(\mathbf{k})$ in Eqs. (29) and (30) is, for the moment, equal to one.

With these equations, we can readily express the KLH (16) in terms of the transformed operators $\tilde{e}_{\mathbf{p} \alpha}, \tilde{h}_{\mathbf{q} \alpha}$, and $\tilde{s}_{l, i}$. To carry out this program, we have to:

i. Substitute Eqs. (29)-(32) in the expression of the Hamiltonian (16),

ii. write the $\tilde{c}_{\mathbf{k} \alpha}$ operators in terms of $\tilde{e}_{\mathbf{p} \alpha}$ and $\tilde{h}_{\mathbf{q} \alpha}$ using Eq. (17),

iii. normal-order these fermionic operators using the canonical anticommutation relations (2),

iv. and contract the $\tilde{s}_{l, i}$ operators corresponding to the same lattice site using the multi-

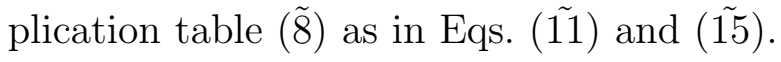

The execution of this program is straightforward but somewhat lengthy, since many terms are generated. To easily identify the procedence of each term in the following discussion, we will symbolically represent Eqs. (29)-(32) by $c^{\dagger}=\tilde{c}^{\dagger}+\delta \tilde{c}^{\dagger}, c=\tilde{c}+\delta \tilde{c}$, and $s=\tilde{s}+\delta \tilde{s}$. 
The kinetic and Kondo parts of $H$ have the structures $c^{\dagger} c$ and $c^{\dagger} c s$ respectively. We will say, for example, that a particular term comes from $\left(\tilde{c}^{\dagger}|\delta \tilde{c}| \tilde{s}\right)$, meaning that it has been generated by executing the steps (ii)-(iv) on the part of the development of $c^{\dagger} c s$ with that structure.

We will now determine $\tilde{T}\left(\mathbf{k}, \mathbf{k}^{\prime}\right)$. Writing $\tilde{T}$ in Eq. (26) with the electron and hole operators (17), we get that $\tilde{T}=\tilde{T}_{1}+\tilde{T}_{2}$, where $\tilde{T}_{1}$ and $\tilde{T}_{2}$ have the structures

$$
\begin{aligned}
& \tilde{T}_{1}=\frac{J}{4 N^{1 / 2}}\left[\tilde{T}(\mathbf{p}, \mathbf{q}) \tilde{e}_{\mathbf{p}}^{\dagger} \tilde{h}_{-\mathbf{q}}^{\dagger} \tilde{s}_{\mathbf{q}-\mathbf{p}}+\tilde{T}(\mathbf{q}, \mathbf{p}) \tilde{h}_{-\mathbf{q}} \tilde{e}_{\mathbf{p}} \tilde{s}_{\mathbf{p}-\mathbf{q}}\right] \\
& \tilde{T}_{2}=\frac{J}{4 N^{1 / 2}}\left[\tilde{T}\left(\mathbf{p}, \mathbf{p}^{\prime}\right) \tilde{e}_{\mathbf{p}}^{\dagger} \tilde{e}_{\mathbf{p}^{\prime}} \tilde{s}_{\mathbf{p}^{\prime}-\mathbf{p}}-\tilde{T}\left(\mathbf{q}, \mathbf{q}^{\prime}\right) \tilde{h}_{-\mathbf{q}^{\prime}}^{\dagger} \tilde{h}_{-\mathbf{q}} \tilde{s}_{\mathbf{q}^{\prime}-\mathbf{q}}\right] .
\end{aligned}
$$

The unitary transformation $\mathrm{e}^{\tilde{T}}$ can be written, to leading order in $J$, as the product of $\mathrm{e}^{\tilde{T}_{2}}$ and $\mathrm{e}^{\tilde{T}_{1}}$. Since $\tilde{T}_{2}|\Phi\rangle=0, \mathrm{e}^{\tilde{T}_{2}}$ does not contribute to change the vacuum state $|\Phi\rangle$, and it can be taken to be the identity without loss of generality:

$$
\tilde{T}\left(\mathbf{p}, \mathbf{p}^{\prime}\right)=\tilde{T}\left(\mathbf{q}, \mathbf{q}^{\prime}\right)=0 \quad \forall \mathbf{p}, \mathbf{p}^{\prime}, \mathbf{q}, \mathbf{q}^{\prime}
$$

The presence of terms of the form $\tilde{e}^{\dagger} \tilde{h}^{\dagger} \tilde{s}$ in $H$ would imply that $|\Phi\rangle$ is not an eigenstate and, hence, not the ground state of the system. In fact, by demanding the cancellation of these terms, we will be able to determine $\tilde{T}(\mathbf{p}, \mathbf{q})$. To leading order in $J$, this condition is given by

$$
(\tilde{22})-\tilde{T}(\mathbf{p},-\mathbf{q})\left(\varepsilon_{\mathbf{p}}-\varepsilon_{\mathbf{q}}\right) \times(\tilde{22})=0,
$$

where the first term comes from $\left(\tilde{c}^{\dagger}|\tilde{c}| \tilde{s}\right)$ and the second from $\left(\tilde{c}^{\dagger} \mid \delta \tilde{c}\right)$ and $\left(\delta \tilde{c}^{\dagger} \mid \tilde{c}\right)$. This condition and Eq. (27) imply that

$$
\tilde{T}(\mathbf{p}, \mathbf{q})=-\tilde{T}(\mathbf{q}, \mathbf{p})=\frac{1}{\varepsilon_{\mathbf{p}}-\varepsilon_{\mathbf{q}}} .
$$

Since $\varepsilon_{\mathbf{p}}>0$ and $\varepsilon_{\mathbf{q}}<0$, the denominator in this equation is always positive. However, when $\mathbf{p}$ and $\mathbf{q}$ are both very close to the Fermi surface (low-energy region), this denominator tends to vanish and $\tilde{T}(\mathbf{p}, \mathbf{q})$ becomes very large. This means that, in this region, higher order terms in $J$ can give important contributions to $\tilde{e}^{\dagger} \tilde{h}^{\dagger} \tilde{s}$. A careful inspection of these terms 
leads to the conclusion that their dominant contribution at low energies comes from $\left(\delta \tilde{c}^{\dagger}|\delta \tilde{c}| \tilde{s}\right)$ and is given by

$$
-\frac{3 J^{2}}{16}\left[\frac{1}{N} \sum_{\mathbf{p}^{\prime}} \tilde{T}\left(\mathbf{p}^{\prime}, \mathbf{q}\right)+\frac{1}{N} \sum_{\mathbf{q}^{\prime}} \tilde{T}\left(\mathbf{p}, \mathbf{q}^{\prime}\right)\right] \tilde{T}(\mathbf{p}, \mathbf{q}) \times(\tilde{22}) .
$$

Assuming a constant density of states $(D=1)$, the sums in this equation can be straightforwardly calculated:

$$
\frac{1}{N} \sum_{\mathbf{p}^{\prime}} \tilde{T}\left(\mathbf{p}^{\prime}, \mathbf{q}\right)=\int_{0}^{1 / 2} \frac{d \varepsilon_{\mathbf{p}^{\prime}}}{\varepsilon_{\mathbf{p}^{\prime}}-\varepsilon_{\mathbf{q}}} \sim \ln \frac{-1}{2 \varepsilon_{\mathbf{q}}}, \quad\left(-\varepsilon_{\mathbf{q}} \ll 1 / 2\right) .
$$

Thus, the terms (38) diverge as $\varepsilon_{\mathbf{p}}$ and $\varepsilon_{\mathbf{q}}$ vanish and, consequently, the cancellation of the contributions to $\tilde{e}^{\dagger} \tilde{h}^{\dagger} \tilde{s}$ is not achieved.

This problematic state-of-affairs can be overcome by introducing a low-energy regulator in Eq. (37), namely,

$$
\tilde{T}(\mathbf{p}, \mathbf{q})=-\tilde{T}(\mathbf{q}, \mathbf{p})=\frac{1}{\varepsilon_{\mathbf{p}}-\varepsilon_{\mathbf{q}}+\tilde{\eta}}
$$

where $\tilde{\eta}$ is a small positive function. Since at high energies this function is neglible compared to $\varepsilon_{\mathbf{p}}$ and $-\varepsilon_{\mathbf{q}}$, it will only play a role at very low energies and, therefore, we can approximately take it as a constant equal to its zero-energy value. Obviously, $\tilde{\eta}$ will be determined by demanding that the sum of all contributions to the terms $\tilde{e}^{\dagger} \tilde{h}^{\dagger} \tilde{s}[(36)$ and (38)] vanish when $\varepsilon_{\mathbf{p}}=\varepsilon_{\mathbf{q}}=0$, namely,

$$
\left[1-\frac{3 J^{2}}{8 \tilde{\eta}} \ln \frac{1}{2 \tilde{\eta}}\right] \times\left.(\tilde{22})\right|_{\varepsilon_{\mathbf{p}}=\varepsilon_{\mathbf{q}}=0}=0 .
$$

Thus, $\tilde{\eta}$ is given, as a function of $J$, by

$$
\tilde{\eta}=\frac{3 J^{2}}{8} \ln \frac{1}{2 \tilde{\eta}}
$$

Substituting (40), we see that the terms (38) are neglegible compared to the first order terms (36) in the high-energy region. Since in this region (40) essentially coincides with (37), Eq. (36) will continue to hold to leading order in $J$ at high energies. In summary, with $\tilde{T}(\mathbf{p}, \mathbf{q})$ and $\tilde{\eta}$ given, respectively, by (40) and (42), we accomplish our objective of cancelling 
the terms $\tilde{e}^{\dagger} \tilde{h}^{\dagger} \tilde{s}$ for all energies and, since $H$ is a hermitian operator, this also implies the cancellation of the terms $\tilde{h} \tilde{e} \tilde{s}$.

To verify that unitarity is indeed preserved by this transformation, we can calculate the canonical properties (2) and (8) in terms of the transformed operators [using Eqs. (29)-(32),

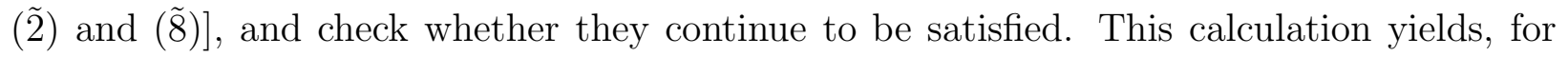
the terms proportional to 1 ,

$$
\begin{aligned}
\left\{c_{\mathbf{k} \alpha}, c_{\mathbf{k}^{\prime} \beta}^{\dagger}\right\} & =[\tilde{\alpha}(\mathbf{k})]^{-2} \delta_{\mathbf{k}, \mathbf{k}^{\prime}} \delta_{\alpha \beta}, \\
s_{0, \mathbf{k}} s_{0, \mathbf{k}^{\prime}} & =\tilde{\sigma}^{-2} \delta_{\mathbf{k},-\mathbf{k}^{\prime}}
\end{aligned}
$$

where

$$
\begin{aligned}
\tilde{\alpha}(\mathbf{k}) & =\left[1+\frac{3 J^{2}}{16 N} \sum_{\mathbf{k}^{\prime}} \tilde{T}^{2}\left(\mathbf{k}^{\prime}, \mathbf{k}\right)\right]^{-1 / 2}=\left[1+\frac{3 J^{2}}{16}\left(\frac{1}{\left|\varepsilon_{\mathbf{k}}\right|+\tilde{\eta}}-\frac{1}{\left|\varepsilon_{\mathbf{k}}\right|+1 / 2}\right)\right]^{-1 / 2} \\
\tilde{\sigma} & =\left[1+\frac{J^{2}}{N^{2}} \sum_{\mathbf{p}, \mathbf{q}} \tilde{T}^{2}(\mathbf{p}, \mathbf{q})\right]^{-1 / 2}=\left[1+J^{2} \ln \frac{1}{4 \tilde{\eta}}\right]^{-1 / 2} .
\end{aligned}
$$

Since both $\tilde{\alpha}(\mathbf{k})$ and $\tilde{\sigma}$ tend to 1 for small values of $J$, the canonical algebraic properties are essentially preserved. This would certainly not be the case if $\tilde{\eta}=0$ and, thus, the introduction of a regulator is not only necessary to cancel the $\tilde{e}^{\dagger} \tilde{h}^{\dagger} \tilde{s}$ terms at low energies, but also to achieve unitarity. The departure of $\tilde{\sigma}$ from 1 is very small and can be neglected. In the case of $\tilde{\alpha}(\mathbf{k})$, however, this departure is not that small at low energies. If $\mathbf{k}_{F}$ is a wave vector of the Fermi surface, $\tilde{\alpha}\left(\mathbf{k}_{F}\right) \sim 1-1 /\left(4 \ln \frac{1}{2 \tilde{\eta}}\right)$. For small $J$, the second term of this equation is certainly neglegible compared to 1 , but it is large compared to $J$. Thus, to satisfy more accurately the canonical anticommutation relations for the two sets of operators, we will substitute the factor $\tilde{\alpha}(\mathbf{k})$ in Eqs. (29) and (30) (which up till now was set equal to one) by the function (45).

Having determined completely the relationship between the initial and transformed operators [Eqs. (29)-(32), (40), (42), and (45)], we can now proceed to calculate $H$ in terms of $\tilde{e}_{\mathbf{p} \alpha}, \tilde{h}_{\mathbf{q} \alpha}$, and $\tilde{s}_{l, i}$ following the steps (i)-(iv) detailed after Eq. (32). From the many contributions to each particular term, we shall only retain the dominant ones for small $J$. 
Next, we summarize the results obtained, indicating where the dominant contributions stem from:

- $\tilde{H}_{\text {kinetic }}$ : The main contributions to the terms $\tilde{e}^{\dagger} \tilde{e}$ and $\tilde{h}^{\dagger} \tilde{h}$ come from $\left(\tilde{c}^{\dagger} \mid \tilde{c}\right),\left(\delta \tilde{c}^{\dagger} \mid \delta \tilde{c}\right)$, $\left(\tilde{c}^{\dagger}|\delta \tilde{c}| \tilde{s}\right)$, and $\left(\delta \tilde{c}^{\dagger}|\tilde{c}| \tilde{s}\right)$, and are given by

$$
\tilde{H}_{\text {kinetic }}=\sum_{\mathbf{p}, \alpha} \tilde{E}\left(\varepsilon_{\mathbf{p}}\right) \tilde{e}_{\mathbf{p} \alpha}^{\dagger} \tilde{e}_{\mathbf{p} \alpha}+\sum_{\mathbf{q}, \alpha} \tilde{E}\left(-\varepsilon_{\mathbf{q}}\right) \tilde{h}_{\mathbf{q} \alpha}^{\dagger} \tilde{h}_{\mathbf{q} \alpha},
$$

where

$$
\tilde{E}\left(\varepsilon_{\mathbf{p}}\right)=\varepsilon_{\mathbf{p}}-\frac{3 J^{2}}{16 N} \sum_{\mathbf{q}}\left[\left(\varepsilon_{\mathbf{p}} \tilde{\alpha}^{2}(\mathbf{p})-\varepsilon_{\mathbf{q}} \tilde{\alpha}^{2}(\mathbf{q})\right) \tilde{T}(\mathbf{p}, \mathbf{q})-2 \tilde{\alpha}(\mathbf{p}) \tilde{\alpha}(\mathbf{q})\right] \tilde{T}(\mathbf{p}, \mathbf{q}) .
$$

Assuming a constant density of states $(D=1)$, this equation can be developed into

$$
\tilde{E}(\varepsilon)=\varepsilon+\frac{3 J^{2}}{16}\left[\left(1-\frac{3 J^{2}}{16} \frac{1}{\varepsilon+\tilde{\eta}}\right) \ln \frac{\varepsilon+1 / 2}{\varepsilon+\tilde{\eta}}+\frac{\tilde{\eta}}{\varepsilon+\tilde{\eta}}\right],(\varepsilon>0) .
$$

- $\tilde{C}$ : The essential contribution to the terms proportional to 1 comes from normalordering the operator $-\sum_{\mathbf{q}, \alpha} \tilde{E}\left(-\varepsilon_{\mathbf{q}}\right) \tilde{h}_{\mathbf{q} \alpha} \tilde{h}_{\mathbf{q} \alpha}^{\dagger}$, which yields

$$
\tilde{C}=-\sum_{\mathbf{q}} 2 \tilde{E}\left(-\varepsilon_{\mathbf{q}}\right)=E_{\Phi_{0}}-\frac{3 J^{2}}{8} N \ln 2
$$

where $E_{\Phi_{0}}=\sum_{\mathbf{q}} 2 \varepsilon_{\mathbf{q}}=-N / 4$ is the energy of the non-interacting vacuum state $\left|\Phi_{0}\right\rangle$.

- $\tilde{H}_{\text {Kondo }}$ : The terms $\tilde{e}^{\dagger} \tilde{h}^{\dagger} \tilde{s}$ and its hermitian conjugates $\tilde{h} \tilde{e} \tilde{s}$ are obviously absent from $H$, since this was the condition used to determine $\tilde{T}(\mathbf{p}, \mathbf{q})$. For the terms $\tilde{e}^{\dagger} \tilde{e} \tilde{s}$ and $\tilde{h}^{\dagger} \tilde{h} \tilde{s}$, the main contributions come from $\left(\tilde{c}^{\dagger}|\tilde{c}| \tilde{s}\right)$ and are given by

$$
\tilde{H}_{\text {Kondo }}=\tilde{\alpha}(\mathbf{p}) \tilde{\alpha}\left(\mathbf{p}^{\prime}\right) \times(\tilde{20})+\tilde{\alpha}(\mathbf{q}) \tilde{\alpha}\left(\mathbf{q}^{\prime}\right) \times \tilde{21} .
$$

- $\tilde{H}_{R K K Y}$ : The dominant contributions to the terms $\tilde{s}_{l, i} \tilde{s}_{l^{\prime}, j}(i \neq j)$ are of order $J^{2}$ and come from $\left(\delta \tilde{c}^{\dagger} \mid \delta \tilde{c}\right),\left(\tilde{c}^{\dagger}|\delta \tilde{c}| \tilde{s}\right)$, and $\left(\delta \tilde{c}^{\dagger}|\tilde{c}| \tilde{s}\right)$. This RKKY interaction is given by

$$
\tilde{H}_{R K K Y}=\frac{1}{2} \sum_{i \neq j} J_{R K K Y}\left(\mathbf{R}_{i}-\mathbf{R}_{j}\right) \tilde{\mathbf{S}}_{i} \tilde{\mathbf{S}}_{j},
$$


where $\tilde{\mathbf{S}}_{i}$ are the vector operators related to $\tilde{s}_{l, i}$ by Eq. (1/7), and $J_{R K K Y}(\mathbf{R})$ is an inter-site exchange coupling given by

$$
J_{R K K Y}(\mathbf{R})=\frac{J^{2}}{N^{2}} \sum_{\mathbf{p}, \mathbf{q}}\left[\varepsilon_{\mathbf{p}} \tilde{\alpha}^{2}(\mathbf{p}) \tilde{T}(\mathbf{p}, \mathbf{q})-2 \tilde{\alpha}(\mathbf{q}) \tilde{\alpha}(\mathbf{p})\right] \tilde{T}(\mathbf{p}, \mathbf{q}) \cos [(\mathbf{q}-\mathbf{p}) \mathbf{R}] .
$$

or, with the approximation $\tilde{\alpha}(\mathbf{k}) \sim 1$,

$$
J_{R K K Y}(\mathbf{R})=\frac{J^{2}}{\Gamma^{2}} \int d \mathbf{p} d \mathbf{q}\left(\frac{\varepsilon_{\mathbf{p}}}{\varepsilon_{\mathbf{p}}-\varepsilon_{\mathbf{q}}+\tilde{\eta}}-2\right) \frac{\cos [(\mathbf{q}-\mathbf{p}) \mathbf{R}]}{\varepsilon_{\mathbf{p}}-\varepsilon_{\mathbf{q}}+\tilde{\eta}},
$$

where $\Gamma$ is the volume of the first Brillouin zone. It is clear from this equation that, in order to determine $J_{R K K Y}(\mathbf{R})$, it is not enough to know the density of states, but we have to specify the entire function $\varepsilon_{\mathbf{k}}$. In one dimension, however, $W=D=1$ and $\varepsilon_{k}=\varepsilon_{-k}$ imply that $\varepsilon_{k}=a|k| / \pi-1 / 2$ where $a$ is the real lattice constant. This dispersion relation leads to

$$
\begin{aligned}
J_{R K K Y}(R)=\frac{J^{2}}{2} \int_{0}^{1 / 2} d & \int_{0}^{1 / 2} d y\left(\frac{y}{1 / 2+y-x+\tilde{\eta}}-2\right) \times \\
& \times \frac{\cos [(1 / 2+y-x) \pi R / a]+\cos [(1 / 2+y+x) \pi R / a]}{1 / 2+y-x+\tilde{\eta}},
\end{aligned}
$$

where, since the integrals converge as $\tilde{\eta} \rightarrow 0$, we can make the approximation of taking $\tilde{\eta}=0$. From Eq. (54), it is clear that $J_{R K K Y}(\mathbf{R})$ is a long-range, oscillating coupling. Carrying out the integrals (55) we find that, for the one-dimensional case, $J_{R K K Y}(R= \pm a)=0.26 J^{2}$, which shows that the exchange coupling between nearest neighbours is antiferromagnetic.

- $\left(\delta \tilde{c}^{\dagger} \mid \delta \tilde{c}\right),\left(\tilde{c}^{\dagger}|\delta \tilde{c}| \tilde{s}\right)$, and $\left(\delta \tilde{c}^{\dagger}|\tilde{c}| \tilde{s}\right)$ also produce terms of the form $\tilde{c}^{\dagger} \tilde{c} \tilde{s}_{i} \tilde{s}_{j}(i \neq j)$. The $\tilde{e}^{\dagger} \tilde{h}^{\dagger} \tilde{s}_{i} \tilde{s}_{j}$ parts and its hermitian conjugates should be cancelled by the $J^{2}$ terms of $\tilde{T}$ in the same way as the leading orders of this transformation cancel the $\tilde{e}^{\dagger} \tilde{h}^{\dagger} \tilde{s}$ and $\tilde{h} \tilde{e} \tilde{s}$ parts. The remaining terms, $\tilde{e}^{\dagger} \tilde{e} \tilde{s}_{i} \tilde{s}_{j}$ and $\tilde{h}^{\dagger} \tilde{h}_{i} \tilde{s}_{j}$, are associated with the interaction between charge carriers and local moments, and it may seem at first sight that they can be neglected because this interaction is dominated by $\tilde{H}_{\text {Kondo }}$. Nevertheless, in the presence of spin correlations without magnetic order (spin liquid), these terms would 
give leading contributions to the low-energy electronic dispersion relation because the main contribution of the Kondo interaction vanishes $\left(\left\langle\Phi\left|\tilde{s}_{l, i}\right| \Phi\right\rangle=0\right)$. However, since the effect of the spin correlations on the charged excitations will not be considered in this article, we will not include these terms in $H$.

By carefully inspecting the rest of the terms generated by this change of coordinates, it can be shown that their effect is negligible compared to the interactions listed above. Thus, the expression of the KLH in terms of the new canonical operators $\tilde{e}, \tilde{h}$, and $\tilde{s}$ is essentially given by

$$
H=\tilde{C}+\tilde{H}_{\text {kinetic }}+\tilde{H}_{\text {Kondo }}+\tilde{H}_{R K K Y} .
$$

\section{SPIN DYNAMICS, MAGNETIC ORDER, AND ELEMENTARY EXCITATIONS}

On the basis of Eq. (56) we can now address the problem of magnetic order. To completely specify the vacuum state $|\Phi\rangle$, we still have to determine the coefficients $C_{\alpha_{1}, \ldots, \alpha_{N}}$ of Eq. (24) which describe the correlations among the local $\tilde{f}$ spins. Since all the terms of $H$ containing tilded fermionic operators annihilate $|\Phi\rangle$, these correlations arise exclusively as a consequence of the induced RKKY interaction. Thus, to determine the coefficients $C_{\alpha_{1}, \ldots, \alpha_{N}}$ we must demand the minimization of

$$
\left\langle\Phi\left|\tilde{H}_{R K K Y}\right| \Phi\right\rangle=\frac{1}{2} \sum_{i \neq j} J_{R K K Y}\left(\mathbf{R}_{i}-\mathbf{R}_{j}\right)\left\langle\Phi\left|\tilde{\mathbf{S}}_{i} \tilde{\mathbf{S}}_{j}\right| \Phi\right\rangle .
$$

This quantity [which would be zero if $C_{\alpha_{1}, \ldots, \alpha_{N}}$ were random coefficients] is negative, and it represents the energy decrease due to the formation of spin correlations. The total ground state energy is given by

$$
E_{\Phi}=E_{\Phi_{0}}-\frac{3 J^{2}}{8} N \ln 2+\left\langle\Phi\left|\tilde{H}_{R K K Y}\right| \Phi\right\rangle
$$


where $E_{\Phi_{0}}$ is the energy of the non-interacting Fermi sea, and the second and third terms represent the energy decreases due to the direct Kondo interaction and the induced spin correlations respectively.

Thus, within the approach presented in this paper, the determination of the magnetic structure of the vacuum state for small couplings is reduced to finding the ground state of a Heisenberg Hamiltonian with an RKKY coupling given by Eq. (54). This is, of course, a very hard task which we do not intend to undertake in this article. However, it should be noted that the situation corresponding to $\tilde{H}_{R K K Y}$ is fundamentally different from that of a typical Heisenberg Hamiltonian coupling only nearest neighbours, because a long-range, oscillating coupling like (54) will inevitably lead to strong frustration and spin fluctuations, which tend to inhibit magnetic order and to favor the formation of a singlet RVB-like spin liquid, 1 . 1 or an antiferromagnet with strongly quenched local moments.

The magnetic nature of the ground state has been usually attributed to the competition between the Kondo and RKKY interactions, establishing a close parallelism with the discussion of the two-impurity case: 8 For weak couplings, the RKKY interaction (which is proportional to $J^{2}$ ) dominates and the two impurities order magnetically, while for stronger couplings, the formation of a Kondo singlet at each impurity site [with binding energy proportional to $\exp (-1 / D J)]$ is energetically favored and magnetic order is destroyed. Between these two extreme cases, we would have intermediate situations in which the local moments are only partially compensated.

As we mentioned in the introduction, the extension of this approach from the twoimpurity case to the Kondo lattice is very unclear due to the Nozières exhaustion problem. Another objection that can be raised is that this mechanism of forming Kondo singlets to compensate the local moments would not be valid for $J<0$ and, therefore, could not explain why systems with ferromagnetic couplings like $\mathrm{CeAl}_{3} 20$ do not exhibit appreciable magnetic order.

The first and most popular attempt to implement in the Kondo lattice this idea of explaining magnetic order as the result of the competition between the RKKY interaction and 
the Kondo singlet formation was carried out by Doniach, 11 who studied a one-dimensional analog of the KLM (the so-called 'Kondo necklace') in which the conduction electrons are represented by pseudo spins. He took as variational antiferromagnetic trial function, the direct product of local wave functions consisting in a linear combination of the singlet and triplet states that can be formed with the local spins and pseudo spins. As a result, he found that magnetic order was destroyed for $J>W$. Although, as he argued, this should only be understood as a qualitative result, it is quite revealing since it essentially says that, if the formation of Kondo singlets was the mechanism responsible for the destruction of magnetic order, this would take place when $J$ is strong enough to overcome the exhaustion problem, enabling the participation of the entire conduction band in the formation of Kondo singlets.

Another well-known approach to the problem of magnetic order in HF systems is due to Coleman and Andrei21, who claimed that the Kondo scattering of low-energy conduction electrons and local moments could stabilize the spin liquid against an antiferromagnetic state which is supposed to have a rather similar energy. This idea is still being investigated by some authors.22

In contrast to these approaches, we have found in the last section that when the RKKY interaction is explicitly extracted from the Hamiltonian, the Kondo interaction is modified in such a way that it no longer influences the ground state. Thus, as we mentioned, the formation of a spin liquid has to be attributed to the long-range character of the RKKY interaction which produces strong frustration and enhances spin fluctuations in the system. Of course, this picture holds only as long as the ground state $|\Phi\rangle$ remains stable. In fact, we will later find that an electronic instability appears for stronger couplings and, at that point, the Kondo coupling between conduction electrons and local moments will indeed influence the spin correlations.

It should be noted that, since $\tilde{H}_{R K K Y}$ is invariant under the change of $J$ by $-J$, the discussion of magnetism is (before the occurrence of the mentioned instability) the same for both ferro and antiferromagnetic couplings. Finally, we would like to point out that other effects not contemplated in the KLM like the existence of a small f-charge fluctuation could 
also contribute to the stabilization of the spin liquid.

The approach presented in this paper, also leads to a physical interpretation of the elementary excitations of the system and their role in the various observable quantities which is fundamentally different from the most popular interpretations. We have seen in the previous section that the expression of the KLH in terms of electron and hole operators associated with the actual vacuum state is inevitably accompanied by the appearance of an explicit RKKY interaction which leads to the formation of spin correlations. Thus, the physical picture of the system at zero temperature motivated by our formalism is that of a backgroung of spin correlations (a spin liquid) in which two kind of modes can be excited: neutral spin modes associated with deformations of the spin liquid and charged modes corresponding to the excitation of electrons and holes in the system.

The accurate determination of the state of the spin system and its excitations would require finding the ground state and spectrum of $\tilde{H}_{R K K Y}$ which, as we mentioned, is beyond the scope of this paper. Nevertheless, we can still draw an important conlusion without having to solve this Hamiltonian: The thermal breakdown of the spin correlations will produce an entropy increase of the order of $N \ln 2$; since $\tilde{H}_{R K K Y}$ is a very weak, second order interaction, the characteristic temperature $T_{K L}$ associated with the spin correlations will be very small and, therefore, this enormous entropy increase will take place in a very small temperature interval, leading to a huge specific heat of spin origin.

Thus, as we mentioned in the introduction, the formalism developed in this paper supports the thesis of Kagan, Kikoin, and Prokof'ev, 16 who argued that a strong mass renormalization of the low-energy conduction electrons cannot explain the high value and universal character of the entropy measured at $T_{K L}$, concluding that the heavy particles responsible for the low-temperature properties of the Kondo lattice should be neutral fermions of spin origin, as opposed to the charged heavy fermions of the slave-boson 13,14 and variational 15 approaches.

In the next two sections, we study the charged excitations of the system. 


\section{CHARGED EXCITATIONS: PERTURVATIVE MODES.}

Since we are interested in studying the zero-temperature charged modes for a Kondo lattice in the normal state, we shall assume that $|\Phi\rangle$ is a singlet spin liquid, namely,

$$
\mathbf{S}|\Phi\rangle=0
$$

where $\mathbf{S}$ is the generator of spin rotations. The spin rotational symmetry will be very useful to characterize the states as eigenvectors of $\mathbf{S}^{2}$ and $\mathbf{S}^{z}$ with definite eigenvalues. For a general state of the form $V|\Phi\rangle$, where $V$ is a linear combination of products of $\tilde{e}_{\mathbf{p} \alpha}^{\dagger}, \tilde{h}_{\mathbf{q} \alpha}^{\dagger}$, and $\tilde{s}_{l, i}$ operators, we can write $\mathrm{e}^{i \alpha \mathbf{S}} V|\Phi\rangle=\mathrm{e}^{i \alpha \mathbf{S}} V \mathrm{e}^{-i \alpha \mathbf{S}}|\Phi\rangle$, which implies that the action of $\mathbf{S}$ on a state is given by

$$
\mathbf{S} V|\Phi\rangle=[\mathbf{S}, V]|\Phi\rangle
$$

We would like to emphasize that, since the transformation (26) preserves the spin symmetry, the commutation rules of $\mathbf{S}$ with the initial and transformed operators are exactly the same. In particular, Eq. (60) implies that the states $\tilde{e}_{\mathbf{p} \alpha}^{\dagger}|\Phi\rangle$ and $\tilde{s}_{l, \mathbf{k}}|\Phi\rangle$ form, respectively, an $s=1 / 2$ charged doublet and an $s=1$ triplet of spin fluctuations. The fact that $S^{z} \tilde{s}_{l, \mathbf{k}}|\Phi\rangle=$ $l \tilde{s}_{l, \mathbf{k}}|\Phi\rangle$ justifies the notation introduced in Eq. (7) for the $s$ operators.

Aside from the contributions of spin correlations, the dispersion relation corresponding to the states $\tilde{e}_{\mathbf{p} \alpha}^{\dagger}|\Phi\rangle$ and $\tilde{h}_{\mathbf{q} \alpha}^{\dagger}|\Phi\rangle$ is given by Eq. (49) and contains a gap of magnitude $2 \tilde{E}(0)=\tilde{\eta}+3 J^{2} / 16$. This statement, however, does not have any physical significance because these states are not the charged excitations of the system.

For small $J$, the actual charged modes are expected to be slight modifications of the states

$\tilde{e}_{\mathbf{p} \alpha}^{\dagger}|\Phi\rangle$ and $\tilde{h}_{\mathbf{q} \alpha}^{\dagger}|\Phi\rangle$ and, consequently, we will refer to them as perturbative modes. However, as we argued in the introduction in relation to the Nozières exhaustion problem, we should also consider the possibility that the Kondo interaction would produce a strong correlation between low-energy electrons and spin waves, leading to the formation of collective states. These strongly correlated modes will be investigated in the next section. 
When we determined $\mathrm{e}^{\tilde{T}}$ in Section II, we argued that the transformation $\mathrm{e}^{\tilde{T}_{2}}$ [Eq. (34)] did not change $|\Phi\rangle$ and, therefore, could be set equal to the identity. Nevertheless, since $\mathrm{e}^{\tilde{T}_{2}}$ does change the basic operators $\tilde{c}_{\mathbf{k} \alpha}$ and $\tilde{s}_{l, \mathbf{k}}$, we can use the degrees of freedom associated to this transformation to get a new set of canonical operators $\hat{c}_{\mathbf{k} \alpha}$ and $\hat{s}_{l, \mathbf{k}}$ which still satisfy $\hat{e}_{\mathbf{p} \alpha}|\Phi\rangle=\hat{h}_{\mathbf{q} \alpha}|\Phi\rangle=0$ and which are optimal in the sense that $\hat{e}_{\mathbf{p} \alpha}^{\dagger}|\Phi\rangle$ and $\hat{h}_{\mathbf{q} \alpha}^{\dagger}|\Phi\rangle$ are the best possible approximations to the actual charged modes.

In the next section, we will calculate this second transformation $(\hat{T})$, and will find that $\hat{c}_{\mathbf{k} \alpha}, \hat{s}_{l, \mathbf{k}}$ are slight deformations of the tilded operators. Since $\hat{c}_{\mathbf{k} \alpha}, \hat{s}_{l, \mathbf{k}}$ are optimal operators, they should be the basis to construct the actual charged modes. In fact, they will be used in the calculation of the strongly correlated modes. To construct the perturbative modes, however, we can also use the tilded operators because the composition of two small deformations is still a small deformation. In fact, the direct calculation with the tilded operators is more reliable because we avoid the inevitable approximations associated with carrying out a second transformation.

Since no symmetry is broken and no bound states are created at this point, there is no physical reason for the appearance of a gap in the charged spectrum. In fact, we will now calculate the dispersion relation for the perturbative modes to leading order in $J$ neglecting the effect of the spin correlations, showing how the gap is indeed closed in this order. The perturbative modes must have $s=1 / 2$. Due to the spin rotational symmetry, the two states in a spin doublet will have the same energy and, therefore, we can select, for instance, the state with $s^{z}=1 / 2$ to carry out our calculations. Moreover, since the situation considered is symmetric with respect to electrons and holes, we only need to calculate the spectrum corresponding, for example, to the electrons. It can be seen, using Eq. (60), that a small deformation of the state $\tilde{e}_{\mathbf{p} \uparrow}^{\dagger}|\Phi\rangle$ having negative charge, $s^{z}=s=1 / 2$, and wave vector $\mathbf{p}$, has the structure

$$
\left|P E_{\mathbf{p} \uparrow}\right\rangle=\alpha(\mathbf{p})\left[\tilde{e}_{\mathbf{p} \uparrow}^{\dagger}-\frac{J}{4 N^{1 / 2}} \beta_{\mathbf{p}}\left(\mathbf{p}^{\prime}\right)\left(\tilde{e}_{\mathbf{p}^{\prime} \uparrow}^{\dagger} \tilde{s}_{0, \mathbf{p}-\mathbf{p}^{\prime}}+\sqrt{2} \tilde{e}_{\mathbf{p}^{\prime} \downarrow}^{\dagger} \tilde{s}_{1, \mathbf{p}-\mathbf{p}^{\prime}}\right)\right]|\Phi\rangle,
$$

where $P E$ stands for 'perturbative electron', $\beta_{\mathbf{p}}\left(\mathbf{p}^{\prime}\right)$ is an arbitrary variational function, and 
$\alpha(\mathbf{p})$ is a normalization factor given by

$$
\alpha(\mathbf{p})=\left[1+\frac{3 J^{2}}{16 \Gamma} \int d \mathbf{p}^{\prime} \beta_{\mathbf{p}}^{\star}\left(\mathbf{p}^{\prime}\right) \beta_{\mathbf{p}}\left(\mathbf{p}^{\prime}\right)\right]^{-1 / 2} .
$$

The function $\beta_{\mathbf{p}}\left(\mathbf{p}^{\prime}\right)$ should be selected to turn the state (61) into an energy eigenstate. This condition can be implemented by demanding that $\beta_{\mathbf{p}}\left(\mathbf{p}^{\prime}\right)$ be an extreme of the functional $E([\beta], \mathbf{p}) \equiv\left\langle P E_{\mathbf{p} \uparrow}|H| P E_{\mathbf{p} \uparrow}\right\rangle$.

Obviously, to calculate this quantity, we will use the expression of $H$ in terms of the tilded operators [Eq. (56)]. The scalar product in this equation can be developed with Eqs. (201), (8), and (9) into an expression in which only scalar products of the form $\left\langle\Phi\left|\tilde{s}_{l_{1}, i_{1}} \ldots \tilde{s}_{l_{m}, i_{m}}\right| \Phi\right\rangle$ with $i_{1}, \ldots, i_{m}$ corresponding to different lattice sites, leave to be determined. These are precisely the spin correlation functions, and depend on the magnetic structure of $|\Phi\rangle$ arising from the RKKY interaction. As we mentioned, the calculation of these correlations is beyond the scope of this paper and, therefore, its effect on the charged modes of the system will be neglected. Thus, we will calculate these modes assuming a disordered spin lattice, which means that all the spin correlation functions vanish, enabling a simple computation of the scalar products. Since the constant $\tilde{C}$ in Eq. (56) coincides, in this case, with the ground state energy, we will remove it from $H$ assigning, in this way, zero energy to $|\Phi\rangle$. This means that the energy of every state is measured with respect to the vacuum state, and represents, therefore, its excitation energy. Obviously, this energy has to be positive; in fact, the existence of a state with negative energy, would imply an instabily of $|\Phi\rangle$ and a transition to a new phase.

With all these conditions taken into account, the calculation of $E([\beta], \mathbf{p})$ yields

$$
\begin{aligned}
& E([\beta], \mathbf{p})=\tilde{E}\left(\varepsilon_{\mathbf{p}}\right)-\frac{3 J^{2}}{16} \alpha^{2}(\mathbf{p})\left\{\frac{\tilde{\alpha}\left(\varepsilon_{\mathbf{p}}\right)}{\Gamma} \int d \mathbf{p}^{\prime} \tilde{\alpha}\left(\varepsilon_{\mathbf{p}^{\prime}}\right)\left[\beta_{\mathbf{p}}^{\star}\left(\mathbf{p}^{\prime}\right)+\beta_{\mathbf{p}}\left(\mathbf{p}^{\prime}\right)\right]\right. \\
& \left.-\frac{1}{\Gamma} \int d \mathbf{p}^{\prime}\left[\tilde{E}\left(\varepsilon_{\mathbf{p}^{\prime}}\right)-\tilde{E}\left(\varepsilon_{\mathbf{p}}\right)\right] \beta_{\mathbf{p}}^{\star}\left(\mathbf{p}^{\prime}\right) \beta_{\mathbf{p}}\left(\mathbf{p}^{\prime}\right)+\frac{J}{2 \Gamma^{2}} \int d \mathbf{p}_{\mathbf{1}} d \mathbf{p}_{\mathbf{2}} \tilde{\alpha}\left(\varepsilon_{\mathbf{p}_{\mathbf{1}}}\right) \tilde{\alpha}\left(\varepsilon_{\mathbf{p}_{\mathbf{2}}}\right) \beta_{\mathbf{p}}^{\star}\left(\mathbf{p}_{\mathbf{1}}\right) \beta_{\mathbf{p}}\left(\mathbf{p}_{\mathbf{2}}\right)\right\},
\end{aligned}
$$

where $\tilde{\alpha}(\varepsilon)$ and $\tilde{E}(\varepsilon)$ are given by Eqs. (45) and (49) respectively. The last term of this equation is of higher order in $J$ than the others, and can be neglected. 
If the gap is indeed closed, $E(0) \equiv E\left([\beta], \mathbf{p}_{F}\right)$ (where $\mathbf{p}_{F}$ is a wave vector of the Fermi surface) must vanish. This quantity can be written as $E(0)=\tilde{E}(0)-\Lambda$, where $\Lambda$ represents the energy decrease due to the Kondo interaction, and is given by

$$
\Lambda=\frac{3 J^{2} \alpha^{2}\left(\mathbf{p}_{F}\right)}{16 \Gamma} \int d \mathbf{p}\left\{\tilde{\alpha}(0) \tilde{\alpha}\left(\varepsilon_{\mathbf{p}}\right)\left[\beta^{\star}(\mathbf{p})+\beta(\mathbf{p})\right]-\left[\tilde{E}\left(\varepsilon_{\mathbf{p}}\right)-\tilde{E}(0)\right] \beta^{\star}(\mathbf{p}) \beta(\mathbf{p})\right\},
$$

where $\beta(\mathbf{p}) \equiv \beta_{\mathbf{p}_{F}}(\mathbf{p})$. Functionally differentiating with respect to $\beta(\mathbf{p})$ in this equation, we find that the condition of extreme of $E(0)$ is given by

$$
\beta(\mathbf{p})=\frac{\tilde{\alpha}(0) \tilde{\alpha}\left(\varepsilon_{\mathbf{p}}\right)}{\tilde{E}\left(\varepsilon_{\mathbf{p}}\right)-\tilde{E}(0)+\Lambda},
$$

and substituting this expression in Eq. (64), we obtain the following equation for $\Lambda$ :

$$
\Lambda=\frac{3 J^{2}}{16} \tilde{\alpha}^{2}(0) \int_{0}^{1 / 2} d \varepsilon \frac{\tilde{\alpha}^{2}(\varepsilon)}{\tilde{E}(\varepsilon)-\tilde{E}(0)+\Lambda} .
$$

As we already mentioned, $\tilde{\alpha}(\varepsilon)$ can be substituted, to leading order in J, by 1. Also, since $\tilde{E}(\varepsilon)-\tilde{E}(0)$ vanishes at $\varepsilon=0$, we can approximate it by $\varepsilon$. Thus, with the approximations

$$
\tilde{\alpha}(\varepsilon) \sim 1, \quad \tilde{E}(\varepsilon) \sim \tilde{E}(0)+\varepsilon
$$

Eq. (66) is turned into

$$
\Lambda=\frac{3 J^{2}}{16} \ln \frac{1}{2 \Lambda}
$$

which implies that $\Lambda=\tilde{\eta} / 2+O\left(J^{2}\right)$. Since $\tilde{E}(0)=\tilde{\eta} / 2+3 J^{2} / 32$, this proves the cancellation of the leading contributions to $E(0)$. We will not verify the cancellation of the $J^{2}$ terms because, since these are lower order contributions, to accurately calculate them, we should have to control all the approximations that have enabled us to carry out our computations analytically. Since, as we already mentioned, there is no physical reason for the formation of a gap, we will assume that the $J^{2}$ contributions cancel as well.

Finally, we would like to point out that if the spin correlations were considered, we should include in $H$ the terms $\tilde{e}^{\dagger} \tilde{e} \tilde{s}_{i} \tilde{s}_{j}$ and $\tilde{h}^{\dagger} \tilde{h}_{i} \tilde{s}_{j}(i \neq j)$ that we mentioned at the end of Section II because, in this case, they give leading contributions to $E(0)$ which compete with those steming from the kinetic and Kondo terms. 


\section{SECOND TRANSFORMATION, STRONGLY CORRELATED CHARGED MODES, AND VACUUM INSTABILITY}

In this section, we shall investigate the possible formation of strongly correlated charged modes. As we already argued in Section IV (and will become even more clear at the end of this section), these modes should be written in terms of optimal fermionic operators representing the best possible approximations to the actual (perturbative) charged excitations. Thus, we will start by carrying out a second unitary transformation to determine these operators, and will find the expression of the Hamiltonian in terms of them. Then, with these building blocks, we will construct the strongly correlated charged modes and will discuss their nature in the case of ferro and antiferromagnetic couplings. Finally, we will show that, for sufficiently strong couplings, these modes condensate in the vacuum state leading to an instability.

In analogy with the notation $(\tilde{\mathrm{x}}),(\hat{\mathrm{x}})$ will denote the equation equivalent to $(\mathrm{x})$ for the optimal operators $\hat{c}_{\mathbf{k} \alpha}, \hat{s}_{l, \mathbf{k}}$. Since we want to continue preserving all the symmetries of $H$, the second unitary transformation $\hat{T}$ that we will now determine must have the structure (26), and the relations between the tilded and hatted operators will be given by Eqs. (20)-

(32) with $\hat{\alpha}(\mathbf{k})$ equal, for the moment, to one. To ensure that $\hat{e}_{\mathbf{p} \alpha}|\Phi\rangle=\hat{h}_{\mathbf{q} \alpha}|\Phi\rangle=0$, e $\mathrm{e}^{\hat{T}}$ must leave $|\Phi\rangle$ invariant, which requires that $\hat{T}(\mathbf{p}, \mathbf{q})=\hat{T}(\mathbf{q}, \mathbf{p})=0$.

We shall take as starting point in the following discussion, the expression (56) of $H$ in terms of the tilded operators with the approximations (67). The use of these approximations in the calculation of quantities in which the dominant contributions cancel will be justified. The main effect of the Kondo interaction is, obviously, to renormalize the charged excitations. Since $\hat{e}_{\mathbf{p} \alpha}^{\dagger}|\Phi\rangle$ and $\hat{h}_{\mathbf{q} \alpha}^{\dagger}|\Phi\rangle$ are optimal approximations to the perturbative charged modes, they will include these renormalization effects and, therefore, they will be subject only to a small residual Kondo interaction. Thus, when we write $H$ in terms of the hatted operators, the bulk of the Kondo interaction should disappear. In analogy with equation (36), the condition for the cancellation of the terms $\hat{e}^{\dagger} \hat{e} \hat{s}$ and $\hat{h}^{\dagger} \hat{h} \hat{s}$ to leading order in $J$ is given by 


$$
\hat{T}\left(\mathbf{p}, \mathbf{p}^{\prime}\right)=\frac{1}{\varepsilon_{\mathbf{p}}-\varepsilon_{\mathbf{p}^{\prime}}}, \hat{T}\left(\mathbf{q}, \mathbf{q}^{\prime}\right)=\frac{1}{\varepsilon_{\mathbf{q}}-\varepsilon_{\mathbf{q}^{\prime}}} .
$$

Again, it can be shown that the singularities of these expressions at the points where $\varepsilon_{\mathbf{k}}=\varepsilon_{\mathbf{k}^{\prime}}$ lead to a loss of unitarity. Thus, we need, once more, to introduce a regulator to tame the singularities. In contast to $\varepsilon_{\mathbf{p}}-\varepsilon_{\mathbf{q}}$ [Eq. (37)] which is always positive, $\varepsilon_{\mathbf{p}}-\varepsilon_{\mathbf{p}^{\prime}}$ and $\varepsilon_{\mathbf{q}}-\varepsilon_{\mathbf{q}^{\prime}}$ can have positive and negative values and, therefore, to avoid the vanishing of the denominators of Eq. (69), we should add to them a small imaginary quantity. Thus, the regulated forms of $\hat{T}\left(\mathbf{p}, \mathbf{p}^{\prime}\right)$ and $\hat{T}\left(\mathbf{q}, \mathbf{q}^{\prime}\right)$ can be written as

$$
\begin{gathered}
\hat{T}\left(\mathbf{p}, \mathbf{p}^{\prime}\right)=\frac{1}{2}\left[\frac{1}{\varepsilon_{\mathbf{p}}-\varepsilon_{\mathbf{p}^{\prime}}+i \hat{\eta}}+\frac{1}{\varepsilon_{\mathbf{p}}-\varepsilon_{\mathbf{p}^{\prime}}-i \hat{\eta}}\right]=\frac{\varepsilon_{\mathbf{p}}-\varepsilon_{\mathbf{p}^{\prime}}}{\left(\varepsilon_{\mathbf{p}}-\varepsilon_{\mathbf{p}^{\prime}}\right)^{2}+\hat{\eta}^{2}} \\
\hat{T}\left(\mathbf{q}, \mathbf{q}^{\prime}\right)=\frac{\varepsilon_{\mathbf{q}}-\varepsilon_{\mathbf{q}^{\prime}}}{\left(\varepsilon_{\mathbf{q}}-\varepsilon_{\mathbf{q}^{\prime}}\right)^{2}+\hat{\eta}^{2}},
\end{gathered}
$$

where, to re-establish unitarity for small couplings, the real constant $\hat{\eta}$ should vanish more slowly than $J^{2}$ as $J \rightarrow 0$. Again, as we did with the first transformation in Section II, to attain unitarity to the next-to-leading order in $J$, we should introduce in Eqs. (29) and (30) the factor $\hat{\alpha}(\mathbf{k})$ given by

$$
\hat{\alpha}(\mathbf{k})=\left[1+\frac{3 J^{2}}{16 N} \sum_{\mathbf{k}^{\prime}} \hat{T}^{2}\left(\mathbf{k}^{\prime}, \mathbf{k}\right)\right]^{-1 / 2}=\left[1+\frac{3 J^{2}}{16} \int_{0}^{1 / 2} d x \frac{\left(\left|\varepsilon_{\mathbf{k}}\right|-x\right)^{2}}{\left[\left(\left|\varepsilon_{\mathbf{k}}\right|-x\right)^{2}+\hat{\eta}^{2}\right]^{2}}\right]^{-1 / 2} .
$$

To completely specify this second transformation, we just need to determine $\hat{\eta}$. We saw in the last section that the Kondo interaction lowered the energy of the charged states around the Fermi level; in fact, the equations (65) and (66) which determined the state $\left|P E_{\mathbf{p}_{F} \uparrow}\right\rangle$, are nothing but the conditions for minimizing the energy of this state. Since $\hat{e}_{\mathbf{p}_{F} \uparrow}^{\dagger}|\Phi\rangle$ must be the best possible approximation to $\left|P E_{\mathbf{p}_{F} \uparrow}\right\rangle$, we will determine $\hat{\eta}$ by demanding that its energy be minimized.

If the spin correlations are disregarded, the energy of the states $\hat{e}_{\mathbf{p} \alpha}^{\dagger}|\Phi\rangle$ is given by the coefficient $\hat{E}(\mathbf{p})$ of the kinetic term $\left(\sum_{\mathbf{p} \alpha} \hat{E}(\mathbf{p}) \hat{e}_{\mathbf{p} \alpha}^{\dagger} \hat{e}_{\mathbf{p} \alpha}\right)$ of $H$ expressed in terms of the hatted operators. In particular, $\hat{e}_{\mathbf{p}_{F} \uparrow}^{\dagger}|\Phi\rangle$ has the energy $\hat{E}(0)=\tilde{E}(0)-\hat{\Lambda}$ where $\hat{\Lambda}$ is given, with the approximations (67) for $\tilde{\alpha}(\varepsilon)$ and $\tilde{E}(\varepsilon)$, by 


$$
\hat{\Lambda}=\frac{3 J^{2}}{16} \int_{0}^{1 / 2} d \varepsilon\left(2 \hat{\alpha}(0) \hat{\alpha}(\varepsilon)-\hat{\alpha}^{2}(\varepsilon) \frac{\varepsilon^{2}}{\varepsilon^{2}+\hat{\eta}^{2}}\right) \frac{\varepsilon}{\varepsilon^{2}+\hat{\eta}^{2}}
$$

where the function $\hat{\alpha}(\varepsilon)$ is given by Eq. (72). After some calculations, it can be shown that the condition of extreme of $\hat{\Lambda}(d \hat{\Lambda} / d \hat{\eta}=0)$ leads to the following equation for $\hat{\eta}$ :

$$
\hat{\eta}=\frac{3 \pi J^{2}}{64} \ln \frac{1}{2 \hat{\eta}}
$$

and that the corresponding (maximum) value of $\hat{\Lambda}$ is given, to order $J^{2}$, by

$$
\hat{\Lambda}=\frac{3 J^{2}}{16}\left(\ln \frac{1}{2 \hat{\eta}}-\frac{1}{2}\right) .
$$

$\hat{E}(0)$ can also be written as $\hat{E}(0)=E(0)+\Lambda-\hat{\Lambda}=\Lambda-\hat{\Lambda}$. Since the dominant parts of $\Lambda$ and $\hat{\Lambda}$ cancel in this equation, $\hat{E}(0)$ will be proportional to $J^{2}$ and it seems that, to calculate the coefficient of proportionality, an accurate computation controlling all the approximations [like (67)] would be needed. However, although these approximations modify the order $J^{2}$ of the energy decreases $\Lambda$ and $\hat{\Lambda}$ corresponding to the states $\left|P E_{\mathbf{p}_{F} \uparrow}\right\rangle$ and $\hat{e}_{\mathbf{p}_{F} \uparrow}^{\dagger}|\Phi\rangle$, their effect on these two states must be very similar and, therefore, they should not essentially change $\hat{E}(0)=\Lambda-\hat{\Lambda}$. Thus, by substituting in this equation the values of $\Lambda$ and $\hat{\Lambda}$ calculated with the same approximations in Eqs. (68) and (75), we should get an estimate of $\hat{E}(0)$. The result obtained in this way is

$$
\hat{E}(0)=\Lambda-\hat{\Lambda}=\frac{3 J^{2}}{16}\left(\ln \frac{\pi}{4}+\frac{1}{2}\right)=0.048 J^{2}
$$

Having determined $\hat{\eta}$ [Eq. (74)], the relations ( $(29)-(32)$ between the tilded and hatted operators are completely specified. In analogy with what we did in Section II with the first transformation, by substituting these relations in Eq. (56), normal-ordering the hatted operators corresponding to electrons and holes, and contracting the operators $\hat{s}_{l, i}$ at the same lattice sites, we obtain the expression of $H$ in terms of the optimal coordinates $\hat{e}_{\mathbf{p} \alpha}, \hat{h}_{\mathbf{q} \alpha}$, and $\hat{s}_{l, \mathbf{k}}$. By carefully examining all the terms generated in this process, we can discriminate the ones which are dominant for small $J$. After this analysis, we find that the new expression of $H$ is essentially given by 


$$
H=\tilde{C}+\hat{H}_{\text {kinetic }}+\hat{H}_{\text {Kondo }}+\hat{H}_{R K K Y}
$$

where

$$
\begin{aligned}
\hat{H}_{\text {kinetic }} & =\sum_{\mathbf{p}, \alpha} \hat{E}\left(\varepsilon_{\mathbf{p}}\right) \hat{e}_{\mathbf{p} \alpha}^{\dagger} \hat{e}_{\mathbf{p} \alpha}+\sum_{\mathbf{q}, \alpha} \hat{E}\left(-\varepsilon_{\mathbf{q}}\right) \hat{h}_{\mathbf{q} \alpha}^{\dagger} \hat{h}_{\mathbf{q} \alpha} \text { with } \hat{E}(\varepsilon) \sim \hat{E}(0)+\varepsilon, \\
\hat{H}_{\text {Kondo }} & =\frac{\hat{\eta}^{2}}{\left(\varepsilon_{\mathbf{p}}-\varepsilon_{\mathbf{p}^{\prime}}\right)^{2}+\hat{\eta}^{2}} \times(\hat{20})+\frac{\hat{\eta}^{2}}{\left(\varepsilon_{\mathbf{q}}-\varepsilon_{\mathbf{q}^{\prime}}\right)^{2}+\hat{\eta}^{2}} \times(\hat{21}) \\
\hat{H}_{R K K Y} & =\frac{1}{2} \sum_{i \neq j} J_{R K K Y}\left(\mathbf{R}_{i}-\mathbf{R}_{j}\right) \hat{\mathbf{S}}_{i} \hat{\mathbf{S}}_{j} .
\end{aligned}
$$

Thus, as expected, the main effects of the second transformation are to strongly reduce the gap in the kinetic term and to substitute the Kondo coupling by a residual Kondo interaction which is effective only between electrons or holes having very similar energies. In contrast to what happened with the first transformation, the technical reason for the appearance of this residual Kondo interaction is that, in this case, the contributions of higher order terms are not important and cannot cancel the Kondo coupling in the regions where $\varepsilon_{\mathbf{k}} \sim \varepsilon_{\mathbf{k}^{\prime}}$

Armed with this (optimal) expression of $H$, we will now look for strongly correlated charged states, namely, we will investigate the possibility that an electron or a hole combines with a wave of spin fluctuations forming a collective state. Again, since the situation is the same for positively and negatively charged modes, we will only need to investigate, for example, the last ones. The structure of such a correlated state having negative charge, wave vector $\mathbf{k}$ and $s=s^{z}=1 / 2$ must be

$$
\left|S C E_{\mathbf{k}, 1 / 2}\right\rangle=N^{-1 / 2} A B(\mathbf{p})\left(\hat{e}_{\mathbf{p} \uparrow}^{\dagger} \hat{s}_{0, \mathbf{k}-\mathbf{p}}+\sqrt{2} \hat{e}_{\mathbf{p} \downarrow}^{\dagger} \hat{s}_{1, \mathbf{k}-\mathbf{p}}\right)|\Phi\rangle
$$

where $S C E$ stands for 'strongly correlated electron', $B(\mathbf{p})$ is a variational function, and $A$ is a normalization factor given by

$$
A=\left[\frac{3}{\Gamma} \int d \mathbf{p} B^{\star}(\mathbf{p}) B(\mathbf{p})\right]^{-1 / 2} .
$$

Neglecting the spin correlations, the energy of this state, measured with respect to the ground state energy, is given by 


$$
E_{S C, 1 / 2}[B]=A^{2}\left[\frac{3}{\Gamma} \int d \mathbf{p} \hat{E}\left(\varepsilon_{\mathbf{p}}\right) B^{\star}(\mathbf{p}) B(\mathbf{p})-\frac{3}{2 \Gamma^{2}} \int d \mathbf{p} d \mathbf{p}^{\prime} B^{\star}\left(\mathbf{p}^{\prime}\right) \hat{J}\left(\varepsilon_{\mathbf{p}^{\prime}}, \varepsilon_{\mathbf{p}}\right) B(\mathbf{p})\right]
$$

where $\hat{J}\left(\varepsilon_{1}, \varepsilon_{2}\right)=J \hat{\eta}^{2} /\left[\left(\varepsilon_{1}-\varepsilon_{2}\right)^{2}+\hat{\eta}^{2}\right]$ is the residual Kondo coupling of Eq. (79).

Obviously, the variational function $B(\mathbf{p})$ will be determined by demanding that $E_{S C, 1 / 2}$ is minimized. This condition is given by

$$
\left(\hat{E}\left(\varepsilon_{\mathbf{p}}\right)-E_{S C, 1 / 2}\right) B(\mathbf{p})-\frac{1}{2 \Gamma} \int d \mathbf{p}^{\prime} \hat{J}\left(\varepsilon_{\mathbf{p}}, \varepsilon_{\mathbf{p}^{\prime}}\right) B\left(\mathbf{p}^{\prime}\right)=0
$$

From Eqs. (83) and (84), we should be able to calculate $E_{S C, 1 / 2}$ and $B(\mathbf{p})$. It should be noted, however, that, to minimize $E_{S C, 1 / 2}$, the kinetic part in Eq. (83) must be kept small, which requires that $B(\mathbf{p})$ have all its weight concentrated in a very narrow layer around the Fermi level. Since the residual Kondo coupling is essentially constant and equal to $J$ in this region, we will make the approximation of substituting in Eq. (84) the function $\hat{J}\left(\varepsilon_{\mathbf{p}}, \varepsilon_{\mathbf{p}^{\prime}}\right)$ by $J$, and will prove later on that this is indeed a valid approximation. Thus, from Eq. (84) we have

$$
B(\mathbf{p})=\frac{Z}{\hat{E}\left(\varepsilon_{\mathbf{p}}\right)-E_{S C, 1 / 2}}, Z=\frac{J}{2 \Gamma} \int d \mathbf{p} B(\mathbf{p}) .
$$

Substituting the first of these equations into the second one, we get the following condition for $E_{S C, 1 / 2}$ :

$$
1=\frac{J}{2} \int_{0}^{1 / 2} \frac{d \varepsilon}{\varepsilon+\hat{E}(0)-E_{S C, 1 / 2}} .
$$

This equation has a solution only for antiferromagnetic couplings $(J>0)$, and it is given by

$$
E_{S C, 1 / 2}=\hat{E}(0)-\Omega_{1 / 2}, \Omega_{1 / 2}=\frac{\mathrm{e}^{-2 / J}}{2} .
$$

For ferromagnetic couplings, the local moments and the spin of the conduction electrons will tend to align parallel to each other and, if a collective state is formed, it should have the structure

$$
\left|S C E_{\mathbf{k}, 3 / 2}\right\rangle=N^{-1 / 2} C D(\mathbf{p}) \hat{e}_{\mathbf{p} \uparrow}^{\dagger} \hat{s}_{1, \mathbf{k}-\mathbf{p}}|\Phi\rangle
$$


which is the general form of a state composed of a charge carrier and a spin wave having wave vector $\mathbf{k}$, negative charge, and $s=s^{z}=3 / 2$. Again, $D(\mathbf{p})$ is a variational function and $C$ a normalization factor. The energy of this state in the absence of spin correlations is given by

$$
E_{S C, 3 / 2}[D]=C^{2}\left[\frac{1}{\Gamma} \int d \mathbf{p} \hat{E}\left(\varepsilon_{\mathbf{p}}\right) D^{\star}(\mathbf{p}) D(\mathbf{p})+\frac{1}{4 \Gamma^{2}} \int d \mathbf{p} d \mathbf{p}^{\prime} D^{\star}\left(\mathbf{p}^{\prime}\right) \hat{J}\left(\varepsilon_{\mathbf{p}^{\prime}}, \varepsilon_{\mathbf{p}}\right) D(\mathbf{p})\right] .
$$

Following the same steps used to estimate $E_{S C, 1 / 2}$, we arrive to an equation for $E_{S C, 3 / 2}$,

$$
1=\frac{-J}{4} \int_{0}^{1 / 2} \frac{d \varepsilon}{\varepsilon+\hat{E}(0)-E_{S C, 3 / 2}}
$$

which has solution only for ferromagnetic couplings and it is given by

$$
E_{S C, 3 / 2}=\hat{E}(0)-\Omega_{3 / 2}, \quad \Omega_{3 / 2}=\frac{\mathrm{e}^{4 / J}}{2}
$$

Thus, summarizing, we have found that in ferro and antiferromagnetic Kondo lattices there is a tendency to form collective states of spin $3 / 2$ and $1 / 2$ respectively, and that, for couplings with the same strength, the formation of these states is much more favored in the antiferromagnetic case $\left(\Omega_{1 / 2} \gg \Omega_{3 / 2}\right)$.

If $\Omega$ stands for either $\Omega_{1 / 2}$ or $\Omega_{3 / 2}$, the probability of finding in a SC mode an electron with wave vector $\mathbf{p}$ in the layer $\varepsilon \leq \varepsilon_{\mathbf{p}} \leq \varepsilon+d \varepsilon$ is $P(\varepsilon) d \varepsilon$, where $P(\varepsilon) \simeq \Omega /(\varepsilon+\Omega)^{2}$. This means, for instance, that $50 \%$ of the electrons in these modes have their wave vectors in the layer $0 \leq \varepsilon_{\mathbf{p}} \leq \Omega$. Thus, if $\Omega \ll \hat{E}(0)$, the $\mathrm{SC}$ modes cannot form because it is energetically more favorable for the electrons in this layer to remain in the PE modes. However, if $\Omega$ is smaller but of the order of $\hat{E}(0)$, the low-energy electrons are energetically allowed to participate in the formation of both $\mathrm{PE}$ and SCE and this will result in a very strong renormalization of the electron (and hole) masses around the Fermi level. Finally, if $\Omega>\hat{E}(0)$, the energy of the SCE will become negative, which means that these states condensate in the ground state producing an instability and the transition to a new phase.

It should be remembered that, to simplify the calculations, we took a constant density of states $D=1$. However, in a real band $D(\varepsilon)$ vanishes at the edges and it is enhanced in 
the middle of the band (Fermi level). Since, as we have already mentioned, the SCE are composed of very soft electrons, only the density of states at the Fermi level $\left(D_{F}\right)$ plays a role in their formation. Thus, more realistic expressions of $\Omega$ are

$$
\Omega_{1 / 2}=\frac{\mathrm{e}^{-2 / J D_{F}}}{2}, \Omega_{3 / 2}=\frac{\mathrm{e}^{4 / J D_{F}}}{2}
$$

Since the value of $\Omega$ depends very strongly on the exponents of these expressions, it is very important to use the correct value of $D_{F}$ when we try to estimate $J_{c}$, namely, the value of $J$ at the phase transition point $(\Omega=\hat{E}(0))$. For example, in the antiferromagnetic case we have (in units of $W) J_{c}\left(D_{F}=1.3\right)=0.34, J_{c}\left(D_{F}=1.5\right)=0.27$, and $J_{c}\left(D_{F}=2\right)=0.17$.

At this point, we would like to show, as we promised, that the approximation $\hat{J}\left(\varepsilon_{\mathbf{p}}, \varepsilon_{\mathbf{p}^{\prime}}\right) \sim$ $J$ used to determine $E_{S C}$ is indeed justified: The average value of the residual Kondo coupling $\hat{J}\left(\varepsilon_{1}, \varepsilon_{2}\right)$ in a SCE state is given by

$$
\langle\hat{J}\rangle=\int_{0}^{1 / 2} \int_{0}^{1 / 2} d \varepsilon_{1} d \varepsilon_{2} P\left(\varepsilon_{2}\right) \hat{J}\left(\varepsilon_{1}, \varepsilon_{2}\right) P\left(\varepsilon_{1}\right)
$$

Substituting the expressions for $P(\varepsilon)$ and $\hat{J}\left(\varepsilon_{1}, \varepsilon_{2}\right)$, this equation can be developed into

$$
\frac{\langle\hat{J}\rangle}{J}=1-2 \int_{0}^{\frac{1}{2 \Omega}} \frac{d x}{x^{2}+\hat{\eta}^{2} / \Omega^{2}}\left(\frac{x+2}{x+1}-\frac{2}{x} \ln (x+1)\right) .
$$

Since the second term of this equation is small when $\hat{\eta} / \Omega \gg 1$ (the actual physical case), $\langle\hat{J}\rangle \sim J$, and our approximation is justified. For example, in the antiferromagnetic case with $D_{F}=1.5$ we have, at $J=J_{c}, \hat{\eta} / \Omega=8.6$ and $\langle\hat{J}\rangle=0.81 J$.

It is interesting to note that in our calculation of the SCE starting from the Hamiltonian (56), we have reduced the minimum energy $\tilde{E}(0)$ of the states $\tilde{e}_{\mathbf{p} \alpha}^{\dagger}|\Phi\rangle$ in two steps: First finding optimal operators $\hat{e}_{\mathbf{p} \alpha}^{\dagger}$ which strongly reduced the minimum energy from $\tilde{E}(0)$ to $\hat{E}(0)$ using the high-energy tail of the Kondo coupling and, second, constructing with these operators SC modes which further reduced the energy using the low-energy part of the Kondo coupling. This makes it very clear why we needed to find the optimal operators to construct the SCE: If we would have built these modes with the operators $\tilde{e}_{\mathbf{p} \alpha}^{\dagger}$ and $\tilde{s}_{l, \mathbf{k}}$, we would not have cacelled the high-energy tail of the Kondo coupling, and the resulting states 
could not be considered as proper states because they would continue interacting strongly with the lattice of local moments.

As we mentioned, we have always neglected the influence of the spin correlations on the charged modes. This influence is actually expected to be important in the quantitative description of the SC modes. At first sight, it seems that these correlations would tend to inhibit the formations of SCE because an additional energy (proportional to $J^{2}$ ) would be needed to excite the spin waves present in these modes; however, the changes in the scalar product introduced by the spin correlations would also modify the contribution of the Kondo interaction to the energy and the overall effect is hard to predict.

The most important challenge posed by this article is the description and characterization of the new phase. Obviously, since we know experimentally that the instabilities in HF systems are associated with antiferromagnetim and superconductivity, it is important to elucidate whether or not the instability that we have described is associated with the onset of superconductivity. In any case, it is clear that the condensation in the ground state of collective charged states will dramatically change the diamagnetic and transport properties of the system. It should also be noted that, since in these condensated states, spin waves strongly couple to conduction electrons, the transition to the new phase will be accompanied by changes in the spin correlations. Thus, since we have attributed the high values of the lowtemperature specific heat to the thermal breakdown of soft spin correlations, an important change in the specific heat is expected to occur at the transition point. In fact, since the state of the spin system affects the SC modes and the condensation of these modes alters the spin system, an accurate calculation of the transition point would require a self-consistent calculation involving the two systems.

\section{SUMMARY AND CONCLUSIONS}

In this article, we have assumed that the KLM incorporates the essential physics of HF systems and, investigating this model at zero temperature and half filling with a "variational 
perturbation theory', we have obtained the following physical picture:

- The non-interacting system consists in an electronic Fermi sea with electrons and holes as elementary excitations, plus a completely degenerate (random) spin lattice.

- For small couplings, the charged spectrum remains basically unchanged but an induced RKKY interaction breaks the degeneracy of the spin lattice.

- At this stage, the RKKY interaction is the only one responsible for the spin dynamics. This very long-range, oscillating interaction implies strong frustration and the enhancement of spin fluctuations, which should explain the formation of a spin liquid or the appearance of a weak magnetic order depending on the crystal structure of the compound.

- The very high values of the low-temperature specific heat measured in these systems are attributed to the enormous entropy increase associated to the thermal breakdown of soft, RKKY-induced spin correlations.

- For higher values of the Kondo coupling, the low-energy electrons and holes are energetically allowed to participate in the formation of both uncorrelated and strongly correlated charged modes which results in a very strong renormalization of the lowenergy charged spectrum. The formation of strongly correlated charged modes is much more favored in antiferromagnetic Kondo lattices than in the ferromagnetic ones.

- Beyond a critical coupling $J_{c}$, the strongly correlated modes condensate in the ground state producing an instability and a transition to a new phase. This transition must be accompanied by an important change in the specific heat because the association of spin waves to conduction electrons to form collective states will modify the localized spin system.

The main future challenges posed by this article are the detailed study of the spin correlations, its inclusion in the calculation of the charged modes, and the description and 
characterization of the new phase.

\section{ACKNOWLEDGEMENTS}

We would like to thank Igor Sandalov for providing discussions and very valuable comments. This work has been financed by the DGICYT (Research Project No. PB93-1249) and by the CIRIT (Grant 1995SGR 00039). J.M. Prats acknowledges financial support from a postdoctoral fellowship from the Ministerio de Educacion y Ciencia of Spain. 


\section{REFERENCES}

${ }^{1}$ Z. Fisk, D.W. Hess, C.J. Pethick, D. Pines, J.L. Smith, J.D. Thompson, and J.O. Willis, Science 239, 33 (1988).

${ }^{2}$ G.R. Stewart, Rev. Mod. Phys. 56, 755 (1984).

${ }^{3}$ P.A. Lee, T.M. Rice, J.W. Serene, L.J. Sham, and J.W. Wilkins, Comments Cond. Matt. Phys. 12, 99 (1986).

${ }^{4}$ P. Fulde, J. Keller, and G. Zwicknagl, Solid State Phys. 41, 1 (1988).

${ }^{5}$ J.R. Schrieffer and P.A. Wolff, Phys. Rev. 149, 491 (1966).

${ }^{6}$ G.D. Mahan, Many-particle physics, (Plenum Press, New York, second edition, 1990), chap. 11.

${ }^{7}$ P. Fulde, Electron correlations in molecules and solids (Springer, third edition, 1995), chap. 12 .

${ }^{8}$ B.A. Jones, C.M. Varma, and J.W. Wilkins, Phys. Rev. Lett. 61, 125 (1988).

${ }^{9}$ K. Yosida, Phys. Rev. 147, 223 (1966).

${ }^{10}$ J.W. Wilkins, in Valence Instabilities, ed. P. Wachter and H. Boppart (North Holland, New York, 1982).

${ }^{11}$ S. Doniach, Physica B 91, 231 (1977).

${ }^{12}$ P. Nozières, Ann. Phys. (Paris) 10, 19 (1985).

${ }^{13}$ P. Coleman, Phys. Rev. B 29, 3035 (1984).

${ }^{14}$ A. Millis and P. Lee, Phys. Rev. B 35, 3394 (1986).

15 T.M. Rice and K. Ueda, Phys. Rev. Lett. 55, 995 (1985); Phys. Rev. B 34, 6420 (1986).

${ }^{16}$ Y. Kagan, K.A. Kikoin, and N.V. Prokof'ev, Physica B 182, 201 (1992). 
${ }^{17}$ Y. Kagan, K.A. Kikoin, and N.V. Prokof'ev, Physica B 194, 1171 (1994).

${ }^{18}$ P.W. Anderson, Mater. Res. Bull. 8, 153 (1973)

${ }^{19}$ P.W. Anderson, Frontiers and Borderlines in Many Particle Physics, ed. R. Broglia and R. Schrieffer (Amsterdam, North Holland, 1988).

${ }^{20}$ K. Andres, J.E. Graebner, and H.R. Ott, Phys. Rev. Lett. 35, 1779 (1975).

${ }^{21}$ P. Coleman and N. Andrei, J. Phys.: Condens. Matter 1, 4057 (1989).

${ }^{22}$ K.A. Kikoin, M.N. Kiselev, and A.S. Mishchenko, Physica B 206, 129 (1995). 